Draft Version June 19, 2021

Preprint typeset using $\mathrm{IAT}_{\mathrm{E}} \mathrm{X}$ style emulateapj v. 5/2/11

\title{
SPATIALLY RESOLVED GAS KINEMATICS WITHIN A Ly $\alpha$ NEBULA: EVIDENCE FOR LARGE-SCALE ROTATION
}

\author{
Moire K. M. Prescott ${ }^{1,2}$, Crystal L. Martin ${ }^{2}$, \& Arjun Dey A $^{3,4}$ \\ Draft version June 19, 2021
}

\begin{abstract}
We use spatially extended measurements of Ly $\alpha$ as well as less optically thick emission lines from an $\approx 80 \mathrm{kpc}$ Ly $\alpha$ nebula at $z \approx 1.67$ to assess the role of resonant scattering and to disentangle kinematic signatures from Ly $\alpha$ radiative transfer effects. We find that the Ly $\alpha$, CIV, HeII, and CIII] emission lines all tell a similar story in this system, and that the kinematics are broadly consistent with large-scale rotation. First, the observed surface brightness profiles are similar in extent in all four lines, strongly favoring a picture in which the Ly $\alpha$ photons are produced in situ instead of being resonantly scattered from a central source. Second, we see low kinematic offsets between Ly $\alpha$ and the less optically thick HeII line $\left(\sim 100-200 \mathrm{~km} \mathrm{~s}^{-1}\right)$, providing further support for the argument that the Ly $\alpha$ and other emission lines are all being produced within the spatially extended gas. Finally, the full velocity field of the system shows coherent velocity shear in all emission lines: $\approx 500 \mathrm{~km} \mathrm{~s}^{-1}$ over the central $\approx 50 \mathrm{kpc}$ of the nebula. The kinematic profiles are broadly consistent with large-scale rotation in a gas disk that is at least partially stable against collapse. These observations suggest that the Ly $\alpha$ nebula represents accreting material that is illuminated by an offset, hidden AGN or distributed star formation, and that is undergoing rotation in a clumpy and turbulent gas disk. With an implied mass of $\mathrm{M}(<R=20 \mathrm{kpc}) \sim 3 \times 10^{11} \mathrm{M}_{\odot}$, this system may represent the early formation of a large Milky Way mass galaxy or galaxy group.
\end{abstract}

Subject headings: galaxies: evolution — galaxies: formation — galaxies: high-redshift

\section{INTRODUCTION}

Giant Ly $\alpha$ nebulae (or "Ly $\alpha$ blobs") are signposts of active galaxy formation. The most luminous examples, which exceed $\sim 100 \mathrm{kpc}$ in size and $\sim 10^{44} \mathrm{erg} \mathrm{s}^{-1}$ in Ly $\alpha$ luminosity, are rare and found primarily in large-scale overdensities (e.g., Steidel et al. 2000; Matsuda et al. 2004, 2005; Saito et al. 2006; Prescott et al. 2008; Yang et al. 2009, 2010; Matsuda et al. 2009, 2011; Erb et al. 2011; Prescott et al. 2012a, 2013). These spatially-extended gaseous nebulae often coexist with star-forming galaxies (i.e., Ly $\alpha$-emitting galaxies, Lyman break galaxies, submillimeter galaxies) and obscured AGN (e.g., Chapman et al. 2004; Matsuda et al. 2004; Dev et al. 2005), suggesting these regions are galaxy groups or clusters in formation (e.g., Prescott 2009; Yang et al. 2009; Prescott et al. 2012b). Thus, giant Lya nebulae contain important clues to the dominant physical mechanisms at work during episodes of massive galaxy formation and offer an observational window into the flow and enrichment of gas within the cosmic web.

Many previous observational studies have focused on the potential power sources within $\operatorname{Ly} \alpha$ nebulae (e.g., Chapman et al. 2004; Basu-Zvch \& Scharf 2004; Dev et al. |2005; Geach et al. 2009; Prescott et al. 2009; Webb et al. 2009; Colbert et al.|2011; Steidel et al.|2011; Yang et al. 2011; (Prescott et al. 2012b), on their po-

\footnotetext{
${ }^{1}$ Dark Cosmology Centre, Niels Bohr Institute, University of Copenhagen, Juliane Maries Vej 30, 2100 Copenhagen , Denmark; mkmprescott@dark-cosmology.dk

2 Department of Physics, Broida Hall, Mail Code 9530, University of California, Santa Barbara, CA 93106, USA

${ }^{3}$ National Optical Astronomy Observatory, 950 North Cherry Avenue, Tucson, AZ 85719, USA

${ }^{4}$ Radcliffe Institute for Advanced Study, Byerly Hall, Harvard University, 10 Garden Street, Cambridge, MA 02138, USA
}

larization properties (Prescott et al. 2011; Haves et al. 2011), on the properties of their dust and molecular gas (Chapman et al. 2001; Yang et al. 2012, 2014a), or on their detailed morphology (Prescott et al. 2012b). Theoretical studies have investigated a variety of powering scenarios: shock-heating in galactic superwinds (e.g., Taniguchi \& Shiova 2000; Taniguchi et al. 2001; Mori et al. 2004), gravitational cooling in infalling cold streams (e.g., Haiman et al. 2000; Fardal et al. 2001; Yang et al. 2006; Diikstra \& Loeb 2009; Goerdt et al. 2010; Faucher-Giguère et al. 2010; Rosdahl \& Blaizot 2012), and resonant scattering or photoionization due to emission from AGN or star formation (e.g., Cantalupo et al. 2005; Kollmeier et al. 2010; Zheng et al. 2011; Cen \& Zheng 2013). Two key issues have emerged from these studies of large Ly $\alpha$ nebulae: (a) how much of the $\operatorname{Ly} \alpha$ emission in these systems is scattered over large spatial scales from a central source versus produced in situ within the nebula and (b) what are the underlying kinematics of the gas?

Theoretically, we know that $\operatorname{Ly} \alpha$ photons should be subject to substantial resonant scattering under typical astrophysical conditions. For the neutral hydrogen column densities typical of Lyman limit systems $\left(\sim 10^{20}\right.$ $\mathrm{cm}^{-2}$ ), the optical depth at line center is of order $10^{7}$ (e.g. Verhamme et al. 2006). Resonant scattering leads to double-peaked emission line profiles as $\operatorname{Ly} \alpha$ photons must diffuse into the wings of the line before they are able to escape the system (e.g., Neufeld 1990; Dijkstra et al. 2006; Verhamme et al. 2006). The large number of scatterings also means that the dust content and its distribution within the system can have a profound effect on the emergent line, preferentially suppressing the Ly $\alpha$ equivalent width in the case of a diffuse distribution or boosting 
it in the case of clumpy dust (Neufeld 1990; Hansen \& Oh 2006, but also see Duval et al. 2014; Laursen et al.|2013). At the same time, gas kinematics can profoundly alter the emission line profile of $\operatorname{Ly} \alpha$ as it emerges from the system, with outflows and infall leading to preferential absorption of the blue or red portion of the line, respectively (e.g., Diikstra et al. 2006; Verhamme et al. 2006: Laursen et al. 2009). For sources at high redshift, absorption of the blue side of the Ly $\alpha$ profile by the intergalactic medium also becomes important (Madau 1995). All these effects are encoded in the shape of the Ly $\alpha$ line profile, so deciphering them requires a side-by-side comparison to a non-resonant line, i.e., a tracer that is not susceptible to resonant scattering, but that is detected from the same region.

As the second brightest emission line of Hydrogen after $\operatorname{Ly} \alpha$, the $\mathrm{H} \alpha$ emission line is perhaps the best nonresonant comparison line for this type of study. Unfortunately, most known Ly $\alpha$ nebulae have been found at redshifts where $\mathrm{H} \alpha$ is difficult to observe from the ground. An important exception is a sample of Ly $\alpha$ nebulae selected by Yang et al. (2010) at $z \approx 2.3$. Using follow-up optical/NIR spectroscopy of Ly $\alpha$ as well as non-resonant emission lines (e.g., [O III], $\mathrm{H} \alpha$ ), they investigated the kinematics of eight of the larger Ly $\alpha$ nebula systems from their sample (Yang et al. 2011, 2014b). However, the ground-based NIR spectroscopic observations in these studies yielded high signal-to-noise ratio information only at the position of galaxies embedded within each Ly $\alpha$ nebula system but were not used to investigate non-resonant line emission (if present) from the extended gas within the nebula itself. A similar analysis was carried out using [O III] detections from two embedded galaxies within a Ly $\alpha$ nebula at $z \approx 3.1$ (McLinden et al. 2013), while a recent IFU study detected [OII], [O III], and $\mathrm{H} \alpha$ within the central $\approx 30 \mathrm{kpc}$ of a Ly $\alpha$ nebula at $z=2.38$ (Overzier et al. 2013).

Probing the kinematics and ionization structure of diffuse gas over larger spatial scales and in regions far from any associated bright galaxies requires mapping out emission-line nebulae in both Ly $\alpha$ as well as less optically thick emission lines. In this context, our discovery of a giant Ly $\alpha$ nebula (henceforth referred to as PRG1) with bright, spatially-extended HeII $\lambda 1640$ emission and somewhat weaker metal lines (Prescott et al. 2009, 2012a, 2013) provides a rare opportunity. HeII $\lambda 1640$ ( "H $\alpha$ " for singly ionized Helium) is a non-resonant line but, unlike $\mathrm{H} \alpha$, it is located in the observed optical at these redshifts where ground-based observations are substantially more sensitive than in the NIR. This source therefore allows us to empirically investigate how much the observed Ly $\alpha$ emission is affected by radiative transfer effects and study the kinematics and ionization of the spatially extended gas within a giant Ly $\alpha$ nebula.

We report the observations and reductions in Section 2 and present the reduced spectra, as well as the surface brightness, emission line, and kinematic profiles in Section 3. In Section 4 we discuss the implications of these results, and we conclude in Section 5. A companion paper will use these same data to explore the energetics of the system as well as any spatial variations in the physical conditions (e.g., metallicity, density, ionization parameter) within the extended Ly $\alpha$ nebula.

In this paper, we assume the standard $\Lambda \mathrm{CDM}$ cos- mology $\left(\Omega_{M}=0.3, \Omega_{\Lambda}=0.7, h=0.7\right)$; the angular scale at $z=1.67$ is $8.47 \mathrm{kpc} /{ }^{\prime \prime}$. All magnitudes are in the AB system (Oke 1974).

\section{OBSERVATIONS \& REDUCTIONS}

We obtained deep spectroscopic observations of PRG1 using the Low Resolution Imaging Spectrometer (LRIS; Oke et al. 1995) on the Keck I Telescope during two separate nights on UT 2009 April 25 and UT 2010 May 10. A summary of the observations is given in Table 1 . All observations used the 400/3400 grism on LRIS-Blue and the 400/8500 grating on LRIS-Red. For each run the data were taken in multislit mode to ensure accurate and repeatable pointing. Masks were designed with a long slitlet centered on PRG1 and the remaining slitlets centered on filler targets in the vicinity. The position of the target on the slitmasks was adjusted to ensure LRIS-Blue wavelength coverage blueward of $\sim 3250 \AA$, the wavelength of Ly $\alpha$ at $z \approx 1.67$.

For the 2009 run, the LRIS-Red grating angle was set to achieve full coverage from the D560 dichroic edge to $\sim 10,000 \AA$. The slitlets centered on PRG1 were $\approx 36-$ $44^{\prime \prime}$ in length, the slitlet widths were set to $1^{\prime \prime} 2$, and the data were unbinned. The resulting spectral resolution at the wavelengths of Ly $\alpha$ and HeII was $\sigma \approx 315$ and $215 \mathrm{~km} \mathrm{~s}^{-1}$, respectively. For the 2010 run, we used the D680 dichroic and set the LRIS-Red grating angle to achieve full coverage from the dichroic edge out to $\sim 10,870 \AA$, in order to cover the $[\mathrm{OII}]$ emission line at $z \approx 1.67$. The slitlets centered on PRG1 were $\approx 48-$ $50^{\prime \prime}$ in length, the slitlet widths were $1^{\prime \prime} .5$, and the LRISBlue data were binned by 2 in the spectral direction in order to reduce the effect of readnoise and increase the signal-to-noise ratio. The resulting spectral resolution at the wavelengths of Ly $\alpha$ and HeII was $\sigma \approx 395$ and 317 $\mathrm{km} \mathrm{s}^{-1}$, respectively.

During each run, the target was observed using two slit position angles: $\mathrm{PA}=52^{\circ} .44$, chosen to traverse the longest dimension of the diffuse emission seen in the ground-based $B_{W}$ image, and $\mathrm{PA}=146.0$, chosen to cover both diffuse emission and the compact red source located at the northwest edge of the nebula (Source A; Prescott et al. 2009). The two slit positions are shown overlaid on the broad-band $B_{W}, R$, and $I$-band images of PRG1 in Figure 1. While there was cirrus at sunset during the 2009 run, it cleared quickly and the observations were taken under clear conditions and $0 .{ }^{\prime \prime} 8-1{ }^{\prime \prime} 1$ seeing; during the 2010 run, the conditions were clear with 0. . $7-0$.'9 seeing. Individual exposures were dithered by $\approx 4-7^{\prime \prime}$ in 2009 and $\approx 7^{\prime \prime}$ in 2010 . The total exposure times on LRIS-Blue/LRIS-Red were 5.2/4.8 hrs for $\mathrm{PA}=52.44$ and $4.9 / 4.8$ hrs for $\mathrm{PA}=146.0$ during the $2009 / 2010$ observing runs.

The data were reduced using IRAF and a customized version of the BOGUS reduction package 5 The first step was to apply the overscan correction and multiply the individual frames by the appropriate gain. Flatfield corrections were applied using domeflat and twilight exposures for the LRIS-Blue data and domeflat frames for the LRIS-Red data. Custom bad pixel masks were used to

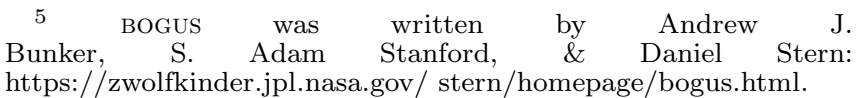


interpolate over bad columns and the script L.A.COSMId was used to identify cosmic rays, both of which were particularly numerous in the 2010 LRIS-Red data. The individual exposures were shifted and stacked to generate the final 2D spectra. The spectral trace was determined using brighter reference objects along the slit, and spectra were extracted both using large apertures centered on the nebula and using a series of narrow apertures spanning the full spatial extent of the diffuse emission. We applied a wavelength calibration determined using HeNeArCdZn comparison lamp exposures and checked the accuracy of the solution using a number of sky lines. Small linear shifts in the wavelength solution relative to the sky lines were measured and removed. Details on the final spectral resolution, spectral range, and wavelength solution are listed in Table 2 .

Our subsequent analysis relies on having an accurate wavelength calibration, particularly in the regions around the Ly $\alpha$ and HeII emission lines. Therefore, as a further check on the accuracy of the wavelength solution, we cross-correlated our calibrated sky spectrum with an independently calibrated sky spectrum from a different program but the same telescope and instrument set-up. The two independently calibrated datasets show no relative shifts between the Ly $\alpha$ and HeII regions of the spectrum.

Flux calibration was applied using standard star exposures of Feige 34 and Wolf 1346 for the 2009 dataset, and Feige 110 and BD+33 2642 for the 2010 dataset 77 The sensitivity functions derived independently for the two datasets agree to within $17 \%$ at the wavelength of Ly $\alpha$ (at $\approx 3250 \AA$, near the atmospheric cut-off) and to within $1-7 \%$ at the wavelengths of CIV, HeII, and CIII]. The larger discrepancy at the location of $\operatorname{Ly} \alpha$ is likely due to the very blue observed wavelength at this redshift $\left(\lambda_{\text {obs }} \approx 3250 \AA\right)$, which means this region of the spectrum is more affected by atmospheric absorption, slit losses, and atmospheric dispersion, as well as larger flat-fielding errors due to the typically red color of flat-field lamps.

\section{RESULTS}

In this section we present the 2D spectra of PRG1 followed by the surface brightness, emission line, and kinematic profiles. Throughout our discussion, a positive velocity offset refers to a redshift of $\operatorname{Ly} \alpha$ relative to the centroid of the HeII $\lambda 1640$ line.

\subsection{Spectra}

Figures 2 3 show the 2D spectra for both the 2009 and 2010 observing runs prior to flux calibration. The Ly $\alpha \lambda 1216, \operatorname{Civ} \lambda 1550$, HeII $\lambda 1640$, and CinI] $\lambda 1909$ (as well as [O II] $\lambda 3727$ in the 2010 data) are clearly detected in both position angles. Continuum emission is detected from the diffuse nebula as well as from several nearby compact sources. The brightest galaxy intersected by the $\mathrm{PA}=146.0$ slit (labeled ' $\mathrm{F}$ ') is a foreground source at $z \approx 0.479$ object, with unambiguous [O II $], \mathrm{H} \beta$, and [O III] emission visible in the LRIS spectroscopy. The second brightest continuum source detected in the $\mathrm{PA}=146^{\circ} 0$

6 L.A.COSMIC was written by Pieter G. van Dokkum: http://www.astro.yale.edu/dokkum/lacosmic/

' KPNO IRS Standard Star Manual; [Massey \& Gronwall] (1990). data, labeled ' $\mathrm{A}$ ' in Figures 2,3, is located at the northwest edge of the diffuse line emission. The 1D spectral extraction at the position of Source A (Figure 4) shows clear Ly $\alpha$ emission and possibly faint HeII emission, likely coming from the nebula rather than from the galaxy itself, but no other strong lines. While there appears to be a hint of emission in the Source A spectrum at the position of $\mathrm{NV}$ at the nebula redshift, the lack of any corresponding emission at CIV or CIII] and the presence of a sky line at exactly the same spectral location leads us to conclude that this is not a real detection of $\mathrm{NV}$. We therefore have no strong spectral constraints on the redshift of Source A, but due to its proximity to the nebula and the lack of continuum emission shortward of Ly $\alpha$, it seems likely that Source A is a galaxy associated with PRG1.

In what follows we combine the two years of data. The derived sensitivity functions between the two runs agree reasonably well, and the HeII flux measurements observed from PRG1 generally agree to within the errors for both position angles. Therefore, we combine the data employing a simple variance-weighted mean after binning the 2009 data by two spectrally to match the 2010 data. We focus in this paper on the restframe UV lines; the [OII] emission will be analyzed in the companion paper on the energetics and physical conditions within PRG1.

\subsection{Surface Brightness Profiles}

Surface brightness profiles are shown for both Ly $\alpha$ and HeII as well as for a stack of the CIV, HeII, and CiII] lines in Figures [5]6] Measured above a surface brightness of $S B_{\text {Ly }} \approx 4.5 \times 10^{-18} \mathrm{erg} \mathrm{s}^{-1} \mathrm{~cm}^{-2} \operatorname{arcsec}^{-2}$, the nebula spans almost $9.5^{\prime \prime} \approx 80 \mathrm{kpc}$ in diameter along the $\mathrm{PA}=52.44$ slit. To compare the relative size of the nebula in each tracer, we measure the diameters containing $50 \%$ and $90 \%$ of the total line luminosity (Table 3). The surface brightness profiles in Ly $\alpha$, the non-resonant HeII line, and the $\mathrm{CIV}+\mathrm{HeII}+\mathrm{CIII}]$ composite are all strikingly similar; for example in terms of half-light diameter, $D_{50}$, the Ly $\alpha$ emission is only slightly more extended than the other rest-frame UV lines, by a factor of $\sim 1.3$. This result is consistent with what we found previously using shallower data (Prescott et al. 2009).

\subsection{Emission Line Profiles}

Figure 7 shows a comparison between the emission line profiles of Ly $\alpha$ and HeII derived from wide extraction apertures chosen to maximize the total signal-to-noise ratio of all four strong lines $\left(7.2^{\prime \prime}=61 \mathrm{kpc}\right.$ and $5.8^{\prime \prime}=49 \mathrm{kpc}$, respectively, for the $\mathrm{PA}=52^{\circ} .44$ and $\mathrm{PA}=146.0$ data). Figures 8] are multi-panel figures showing the same emission line profile comparison but as a function of position along the slit, where the individual panels corresponds to subapertures $\left(5\right.$ pix $\left.\approx 0.67^{\prime \prime}\right)$ spanning the full extent of the nebula. The vertical dashed lines represent the systemic velocity, defined as the centroid of the HeII line at the position where the two slits cross. Our spectral resolution is not sufficient to resolve multiple peaks in the Ly $\alpha$ emission line due to radiative transfer effects, so instead we focus on the centroid offsets of the Ly $\alpha$ line relative to the non-resonant HeII line.

In Figure 10 we plot the velocity offset of Ly $\alpha$ measured in thirteen spatial apertures within the nebula 
(5 pix $\approx 0.67^{\prime \prime}$, with a minimum $S N R=3$ in both Lya and HeII) and compare with similar measurements from other galaxy populations. The typical observed offset between the two lines is $\Delta v \equiv v_{\text {Ly } \alpha}-v_{\text {HeII }} \sim 100-$ $200 \mathrm{~km} \mathrm{~s}^{-1}$, depending on position. This low velocity offset is similar to what has been seen in other Ly $\alpha$ nebulae (Yang et al. 2011; McLinden et al. 2013), however, here we are able to probe the kinematics point-by-point within the extended gas to show that the velocity offset is consistently low across the entire $\sim 80 \mathrm{kpc}$ nebula. The measured velocity offset is less than what is seen in LBGs (Steidel et al. 2010) and more similar to that observed in Ly $\alpha$-emitting galaxies (McLinden et al. 2011; Hashimoto et al. 2013; Guaita et al. 2013; Song et al. 2014).

\subsection{Kinematic Profiles}

In Figures 11,12, we present the velocity and velocity dispersion profiles for Ly $\alpha$ and HeII. Again, we see very small velocity offsets between Ly $\alpha$ and HeII; typically in the range of $\sim 100-200 \mathrm{~km} \mathrm{~s}^{-1}$ to the red. At the same time, the large-scale velocity profile in the $\mathrm{PA}=52^{\circ} .44$ slit shows a coherent velocity gradient $-\sim 500 \mathrm{~km} \mathrm{~s}^{-1}$ over the central $50 \mathrm{kpc}$ of the nebula - while in the $\mathrm{PA}=146.0$ slit, the velocity profile is much shallower. While in most spatial apertures the kinematic offset is to the red, in $\mathrm{PA}=146^{\circ} .0$ on the side of the nebula closest to Source A there is a hint of a reversal, i.e., Ly $\alpha$ is slightly offset to the blue.

The linewidths measured as a function of position are shown in the lower panels of Figures 11]12. Across the $\mathrm{PA}=52^{\circ} .44$ slit, the linewidth profile is quite flat, only marginally resolved in Ly $\alpha$, and unresolved in HeII. On the other hand, in $\mathrm{PA}=146.0$ we see a peak in the linewidth profile that is clearly resolved and spatially coincident in both Ly $\alpha$ and HeII. In later sections, we use these linewidths as a measure of the velocity dispersion as a function of position within the nebula. However, given the spatial resolution of our data, we note that these measurements inevitably includes a contribution from macroscopic velocity gradients, i.e., of order 80 $\mathrm{km} \mathrm{s}^{-1}$ across the $1^{\prime \prime}$ seeing disk for $\mathrm{PA}=52^{\circ} .44$.

\section{DISCUSSION}

Using the results of the previous section, we can now address the questions posed in Section 10 what is the role of resonant scattering in creating the extended Ly $\alpha$ nebula, and what are the kinematics of the gas? We then discuss the implications of these results for the physical model and underlying power source for PRG1.

\subsection{Lyo Optical Depth: A Back-of-the-Envelope Estimate}

We expect that $\operatorname{Ly} \alpha$ will be optically thick under all but the most extreme situations, yet the consistently small velocity offsets we observe between Ly $\alpha$ and the non-resonant HeII line suggest that Ly $\alpha$ is not being substantially affected by complex radiative transfer in PRG1. This implies either that Ly $\alpha$ is optically thin (which we will demonstrate in this section is not likely the case), or that Ly $\alpha$ is produced in situ over an extended area, thereby largely avoiding the effects resonant scattering would impart.
Before discussing our observations in more detail, it is useful to review how optically thin Ly $\alpha$ can be under plausible astrophysical conditions, particularly under the limiting case of a highly ionized medium.

We start by making the assumption of ionization equilibrium:

$$
\begin{array}{r}
\alpha_{B} n_{e} n_{H I I}=\Gamma n_{H I} \\
\Gamma \approx Q \sigma_{p} /\left(4 \pi R^{2}\right)
\end{array}
$$

where $n_{e}$ is the electron number density $\left(\mathrm{cm}^{-3}\right), \mathrm{n}_{H I}$ is the HI number density $\left(\mathrm{cm}^{-3}\right), \mathrm{n}_{H I I}$ is the HII number density $\left(\mathrm{cm}^{-3}\right), \Gamma$ is the photoionization rate (photoionizations/s), $Q$ is the luminosity of ionizing photons (photons/s), $R$ is the radius from ionizing source $(\mathrm{cm})$, $\sigma_{p}$ is the photoionization cross-section $\left(\mathrm{cm}^{2}\right)$, and $\alpha_{B}$ is the Case $\mathrm{B}$ recombination coefficient for $H I$.

The Ly $\alpha$ optical depth is:

$$
\tau_{L y a}=n_{H I} \sigma_{L y a} L
$$

where $\tau_{L y a}$ is the optical depth at line center, $n_{H I}$ is the HI number density $\left(\mathrm{cm}^{-3}\right), \sigma_{L y a}$ is the Lya absorption cross-section $\left(\mathrm{cm}^{2}\right)$, and $L$ is the path through system $(\mathrm{cm})$.

We make the approximation that $\mathrm{H}$ is highly ionized (e.g., in the region around an AGN):

$$
n_{H I I} \sim n_{H} \sim n_{e}
$$

Substituting into equation (1), we obtain the following relation:

$$
\begin{aligned}
n_{H I} & \approx \alpha_{B} n_{H}^{2} / \Gamma \\
& \approx 4 \pi R^{2} \alpha_{B} n_{H}^{2} /\left(Q \sigma_{p}\right)
\end{aligned}
$$

We rewrite this in terms of the ionization parameter $U=Q /\left(4 \pi R^{2} c n_{H}\right)$, where $c$ is the speed of light:

$$
n_{H I} \approx \alpha_{B} n_{H} /\left(c \sigma_{p} U\right)
$$

Combining this relation with equation (3) yields:

$$
\tau_{L y a} \approx \alpha_{B} n_{H} \sigma_{L y a} L /\left(c \sigma_{p} U\right)
$$

We adopt the following values: $\alpha_{B}=2.59 \times 10^{-13}$ $\mathrm{cm}^{3} \mathrm{~s}^{-1}, \sigma_{p}=6.3 \times 10^{-18} \mathrm{~cm}^{2}$ at $1 \mathrm{Ryd}, \sigma_{L y a}=5.9 \times$ $10^{-14} \mathrm{~cm}^{2}$ (Storey \& Hummer 1995; Verner et al. 1996; Osterbrock 1989) $, c=3.00 \times 10^{10} \mathrm{~cm} \mathrm{~s}^{-1}, L=50 \mathrm{kpc}$ and $n_{H}=1.0 \mathrm{~cm}^{-3}$.

Taking a range of ionization parameter values $(U=$ $\left.10^{-3}-1\right)$ results in a Ly $\alpha$ optical depth of:

$$
\tau_{\text {Lya }} \approx 1.2 \times 10^{7}-1.2 \times 10^{4}
$$

in the limit of high ionization.

In order for $\tau_{\text {Lya }}<1$, we would therefore need one of the following to be true:

- $U \gtrsim 10^{4}$, which is many orders of magnitude higher than what is measured for a typical AGN broad line region (Peterson 1997).

- $n_{H} \lesssim 10^{-7}-10^{-4} \mathrm{~cm}^{-3}$, which is much less than the (albeit uncertain) existing measurements of $n_{H}$ 
for Ly $\alpha$ nebulae $\left(\sim 1-30 \mathrm{~cm} \mathrm{~s}^{-3}\right.$; e.g., Dey et al. 2005; Prescott et al. 2009).

- $L \lesssim 5 \times 10^{-3}-5$ pc, i.e., the Ly $\alpha$ is emerging from a very thin skin. This could arise if (a) the Ly $\alpha$ we observe is produced via photoionization within a "blister HII region" illuminated by an offset source, or if (b) Ly $\alpha$ is produced throughout the cloud but all buried Ly $\alpha$ is efficiently extinguished, i.e., by dust.

Thus, even in the highly ionized limit, $\tau_{L y a}>>1$ for most reasonable physical parameters. Given sufficient spectral resolution, we would expect to see evidence for substantial resonant scattering of the $\operatorname{Ly} \alpha$ line in the form of a larger spatial extent and/or intrinsically double peaked, complex profiles with kinematic offsets relative to a non-resonant tracer. At lower spectral resolution, this would translate into broadened Ly $\alpha$ emission lines with offsets in the observed line centroid.

\subsection{Extended Line Emission and the Role of Resonant Scattering}

How much is Ly $\alpha$ being affected by radiative transfer effects relative to the other emission lines, or similarly, how much is Ly $\alpha$ resonant scattering responsible for the large physical extent of the nebula seen in Ly $\alpha$ ? At the spectral resolution of our data, we would expect that any intrinsically complex, multi-peak Ly $\alpha$ profiles to result in the observed Ly $\alpha$ line being broader than a non-resonant tracer. This is consistent with our observation that the Ly $\alpha$ line is broader than HeII in the aperture where both lines are resolved $\left(\sigma_{H e I I} \sim 390 \mathrm{~km} \mathrm{~s}^{-1}\right.$ and $\sigma_{L y \alpha} \sim 570$ $\mathrm{km} \mathrm{s}^{-1}$ for Ly $\alpha$, after correcting for the instrumental resolution; Figure 12. This suggests that, as expected, Ly $\alpha$ is more optically thick and undergoing more complicated radiative transfer than the HeII line.

However, the simple observational fact that a nonresonant line like HeII is seen to be nearly as spatially extended as the Ly $\alpha$ emission is a strong argument that resonant scattering of centrally-produced Ly $\alpha$ is not the primary factor responsible for the large spatial extent of the Ly $\alpha$ emission. A similar observation was made in the case of LABd05, a Ly $\alpha$ nebula at $z \approx 2.7$ that shows diffuse UV continuum emission comparable in extent to the Ly $\alpha$ (Prescott et al. 2012b). In at least these two systems, Ly $\alpha$ scattering is not the main reason we observe a $\sim 100 \mathrm{kpc}$ scale $\mathrm{Ly} \alpha$ nebula. Instead, the Ly $\alpha$ photons are predominantly being produced in situ within the extended gas. At the same time, polarization data from a different Ly $\alpha$ nebula system suggests a significant contribution from scattered Lya emission (SSA22-LAB1; Haves et al. 2011). The prevalence of scattering versus in situ production in Ly $\alpha$ nebulae as a class remains to be quantified, but these few case studies suggest that both mechanisms play a role in producing extended Lya sources.

\subsection{Kinematics within the Spatially Extended Gas}

Ly $\alpha$ radiative transfer modeling indicates that Ly $\alpha$ photons propagating through outflowing or infalling gas should appear redshifted or blueshifted, respectively, relative to the systemic velocity (e.g., Dijkstra et al. 2006; Verhamme et al. 2006). With this in mind, we can ask whether Ly $\alpha$ shows velocity offsets relative to the nonresonant HeII line in PRG1. In general, we see relatively small offsets between the two lines (typically $\sim 100-200$ $\mathrm{km} \mathrm{s}^{-1}$ ), with Ly $\alpha$ usually shifted to the red (suggestive of an outflow). In the case of a simple outflowing shell model, these offsets would correspond to expansion velocities of $\sim 50-100 \mathrm{~km} \mathrm{~s}^{-1}$ (e.g., Verhamme et al. 2006). In addition, a few spatial apertures (located near Source A) show blue-shifts of $\operatorname{Ly} \alpha$ suggestive of mild infall. As we showed in Section [3.3. these velocity offsets are overall lower than what is seen in typical UVselected star-forming galaxies, and comparable or perhaps slightly lower than for Ly $\alpha$-emitting galaxies.

By itself, the low velocity offsets could imply one of several possibilities. The outflow velocities could be intrinsically lower in these systems, or the column density of neutral gas could be low (either globally or due to local patchiness). Alternatively, since the radiative transfer from an extended source of emissivity generically results in observed kinematic offsets that are suppressed relative to the case of a central source (e.g., Verhamme et al. 2006), the low velocity offsets could simply reflect the fact that the $\operatorname{Ly} \alpha$ photons in the $\operatorname{Ly} \alpha$ nebula are being produced over an extended region, rather than being scattered from a central source. In this scenario, the Ly $\alpha$ profiles in each aperture do not actually encode information about the full velocity structure of the system, but instead reflect only small local velocity offsets between the point of emission of the Ly $\alpha$ photons and the final scattering location. For this reason, the Ly $\alpha$ kinematics closely resemble what is measured using a non-resonant line generated within the same region. Combined with the results of the previous section, it seems clear that both the spatial structure and kinematics of the system are consistent with in situ production of Ly $\alpha$ photons in PRG1. From a purely observational perspective, the fact that Ly $\alpha$ traces the non-resonant HeII line so well, suggests that using Ly $\alpha$ alone to do kinematic studies may actually be more reliable in this case than is often assumed.

\subsection{Towards a Physical Model for PRG1: Evidence for Large-scale Rotation}

The deep spectroscopy allows us to probe the kinematics of the diffuse gas using multiple lines with great sensitivity out to large physical scales. A successful physical model for the gas kinematics in PRG1 must be able to explain the following observations: (1) a pronounced monotonic velocity gradient in the $\mathrm{PA}=52^{\circ} .44$ data, with a flattening at large radii, and a relatively flat velocity profile in $\mathrm{PA}=146.0$; (2) a consistently low velocity dispersion in $\mathrm{PA}=52^{\circ} .44\left(\sigma_{L y \alpha} \lesssim 300 \mathrm{~km} \mathrm{~s}^{-1}\right.$, corrected for the instrumental resolution), and (3) a conspicuous resolved peak in the velocity dispersion profiles for both HeII and $\mathrm{Ly} \alpha$ in the $\mathrm{PA}=146^{\circ} 0$ slit, corresponding to $\sigma_{H e I I} \sim 390 \mathrm{~km} \mathrm{~s}^{-1}$ and $\sigma_{L y \alpha} \sim 570 \mathrm{~km} \mathrm{~s}^{-1}$.

It is easiest to understand the velocity profile within the system if the nebula represents gas undergoing rotation. To show this, we construct a toy model of a simple thin disk with six parameters: the offset angle between the $\mathrm{PA}=52^{\circ} .44$ slit and the major axis of the disk $\left(\Theta_{o f f}\right.$, between $-45^{\circ}$ to $\left.45^{\circ}\right)$, the disk inclination $\left(i\right.$, between $0^{\circ}$ and $90^{\circ}$ ), the maximum velocity of the disk ( $V_{\max }$, be- 
tween 0 and $600 \mathrm{~km} \mathrm{~s}^{-1}$ ), the radius at which the disk reaches $V_{\max }\left(R_{\max }\right.$, between 0 and $\left.6^{\prime \prime}\right)$, and the position of the slit crossover relative to the disk center $\left(X_{c}\right.$, $Y_{c}$, within $\pm 2^{\prime \prime}$, where a positive offset in both parameters indicates a slit crossover located to the northwest of the disk center). We use a simple Markov Chain Monte Carlo (MCMC) fitting approach and 100,000 iterations to determine the best fit to the velocity profile in both slits, and estimate the posterior distribution for each parameter (Figure 13). While we have shown that Ly $\alpha$ and HeII show very similar behavior overall, there are small differences, particular in the region around Source A. For the purposes of fitting the velocity profiles, therefore, we use the more reliable HeII line and restrict the fitting to only those apertures within the central $R<3.5^{\prime \prime}$ of the nebula.

The posterior distributions suggest that the velocity profiles agree reasonably well with a thin disk model. In Table 4, we list the $67 \%$ confidence intervals for each parameter, and in Figure 14 we show the range of predicted velocity profiles corresponding to these confidence intervals as well as the predicted profiles for two random draws from the posterior distributions, overplotted on the data. The corresponding disk dynamical masses are of the order of $\mathrm{M}(<\mathrm{R}=20 \mathrm{kpc}) \sim 3 \times 10^{11} \mathrm{M}_{\odot}$. The fact that the major axis of the model disk is roughly aligned with $\mathrm{PA}=52.44$ explains the classic "rotation curve" structure seen in the velocity profile, i.e., the monotonic velocity gradient and the flattening at large radii. Similarly, the $\mathrm{PA}=146.0$ slit lies roughly along the minor axis, explaining the flatter velocity profile. We note that the surface brightness profiles of PRG1 can be reasonably well fit with an exponential profile, consistent with this picture.

The velocity dispersion for a simple rotating disk observed at some inclination angle is a combination of the intrinsic velocity dispersion (e.g., due to turbulence) as well as the smearing of the disk rotation within the slit; the latter component typically leads to an observed peak in the velocity dispersion at the disk center. Our simple thin disk model does not allow for a formal prediction of the velocity dispersion, but the spread between the edges of the slit can be taken as an indication of the velocity dispersion that would be measured simply due to rotation smearing. For reasonable models, we find that the spread in velocity sampled by the slit is only of order 200 $\mathrm{km} \mathrm{s}^{-1}\left(\sigma \lesssim 100 \mathrm{~km} \mathrm{~s}^{-1}\right)$, well below the resolution of our data. Thus, if the kinematics were driven solely by rotation in a thin disk, we would not expect to resolve the lines anywhere across the nebula. In addition, the MCMC fitting analysis strongly prefers a center of rotation that is offset with respect to the velocity dispersion peak by about 1-2" to the northwest. Together, these facts suggest the velocity dispersion peak we observe is not the kinematic center of the system, but rather the result of local kinematics, e.g., an outflow from a galaxy or clump within the nebula. It is possible that PRG1 resembles a scaled up version of the "clumpy disks" seen at $z \approx 2$ (e.g., Förster Schreiber et al. 2009; Newman et al. 2012), which in some cases show a peak in the velocity dispersion that is offset by several kiloparsecs from the disk center due to the presence of a large star-forming clump driving an outflow. In the case of PRG1, this possibility is supported by the presence of a continuum source near the location of the peak velocity dispersion that is visible in recently acquired $H S T$ /WFC3 F140W imaging (Prescott et al., in prep.).

Despite its simplicity, the toy model provides a good representation of the velocity profiles observed in PRG1. To estimate the stability of the proposed disk, we plot the ratio of the velocity dispersion (corrected for the instrumental resolution, $\left.\sigma_{c o r r}\right)$ and the circular velocity $\left(V_{c}\right.$, measured from HeII) as a function of position along the $\mathrm{PA}=52.44$ slit (Figure 15); as the $\mathrm{PA}=52.44$ slit corresponds roughly to the major axis of the disk and the preferred disk inclination is relatively high, we do not apply any corrections for the disk inclination or azimuthal angle within the disk, i.e., we take $V_{c} \approx V_{o b s}$, the observed velocity. Apertures where the measured linewidth is consistent with the instrumental resolution are shown as upper limits $(3 \sigma)$. Using the one aperture along $\mathrm{PA}=146^{\circ}$.0 where both Ly $\alpha$ and HeII are clearly resolved, we compute an approximate "radiative transfer correction" for Ly $\alpha$, i.e., the factor by which the Ly $\alpha$ linewidth should be scaled down in order to match that of the non-resonant HeII line. Under the crude assumption that this factor can be applied across the entire nebula, this approach provides a means of peering below our instrumental resolution limit. The Ly $\alpha$ measurements are then plotted both with and without this "radiative transfer correction." Following Genzel et al. (2014) and assuming a marginally stable disk, we estimate the approximate Toomre Q parameter, a measure of whether the gas will be unstable to collapse and result in subsequent star formation, where $Q_{\text {approx }} \approx(a / 1.4) \times\left(1.0 / f_{\text {gas }}\right) \times \sigma_{\text {corr }} / V_{c}$, with $a$ being a geometric factor with values of $[1.0,1.4,2.0]$ for a Keplerian rotation curve, a flat rotation curve, and a solid-body rotation curve, respectively, and with $f_{g a s}$ being the gas mass fraction. Over most of the nebula, we can only report upper limits on the $\sigma_{c o r r} / V_{c}$, but in regions where the lines are resolved, we estimate that $Q_{\text {approx }}$ is typically greater than $0.67-1.3$, i.e., the critical values below which the gas becomes unstable, even under the assumption of an extremely high gas fraction $\left(f_{\text {gas }}=1\right)$. Thus in most of the apertures where we resolve the Ly $\alpha$ emission line, the nebula appears to be stable against collapse. In a few central apertures as well as at larger radii, however, the gas may be unstable to collapse and subsequent star formation.

Deep IFU observations of Ly $\alpha$ nebula such as PRG1 detecting multiple emission lines will be important for shedding further light on the complex kinematics of these systems, but the suggestion from the data presented here is that the gas in PRG1 is undergoing large-scale rotation in a clumpy, turbulent disk.

\subsection{Implications}

Recent high resolution numerical simulations of Milky Way mass halos $\left(\sim 10^{12} \mathrm{M}_{\odot}\right.$ at $z=0$, which corresponds to $\sim 10^{11} \mathrm{M}_{\odot}$ at $z=3$, assuming the halo growth rate from Neistein et al. 2006) have indicated that newly accreted gas will have high angular momentum, spending 1-2 dynamical times in the outer halo as a "cold flow disk" that extends to many tens of kiloparsecs outside the central galaxy (Stewart et al. 2011, 2013). Although the halo masses of Ly $\alpha$ nebulae are poorly constrained, it is possible that we are seeing a similar phenomenon in Ly $\alpha$ nebulae systems like PRG1 and SSA22-LAB2 
(Martin et al. 2014), i.e., the early formation of a large Milky Way mass galaxy or galaxy group. In the case of PRG1, the rotation period implied by our disk modeling $\left(T_{\text {rot }} \approx 4.9 \times 10^{8} \mathrm{yr}\right.$, assuming $V_{\max } \approx 250 \mathrm{~km} \mathrm{~s}^{-1}$ at $R=20 \mathrm{kpc}$ ) is consistent with the system undergoing a handful of rotations by $z \approx 1.67$ (when the age of the Universe was $\sim 3.9$ Gyr). Our results also motivate further high resolution theoretical work on the angular momentum of cold gas accretion as a function of halo mass, particularly for the higher mass halos thought to host giant Ly $\alpha$ nebulae (e.g., Prescott et al. 2008; Yang et al. 2009, 2010).

At the same time, our data do not favor the idea that gravitational cooling is the dominant powering mechanism responsible for the $\operatorname{Ly} \alpha$ emission in this system. Gravitational infall of lower metallicity gas (i.e., "cold flows") would not be expected to produce such strong HeII emission over such a large spatial extent (Yang et al. 2006; Rosdahl \& Blaizot 2012), and the presence of CIV and CIII] indicates the gas is at least somewhat enriched. In addition, cold flow powered Ly $\alpha$ nebulae are predicted to exhibit Ly $\alpha$ emission line profiles with a dominant blue peak, owing to infall (Faucher-Giguère et al. 2010), whereas in PRG1 we find that whether Ly $\alpha$ is observed to be redshifted or blueshifted relative to HeII depends on the position within the nebula, with most locations showing redshifted $\operatorname{Ly} \alpha$ emission. In addition, there is still debate as to whether gravitational cooling can provide the Ly $\alpha$ luminosities that are typically observed in Ly $\alpha$ nebulae (e.g., Goerdt et al. 2010; Faucher-Giguère et al. 2010; Rosdahl \& Blaizot 2012).

What does seem likely is that the gas reservoir in these regions is being supplied by recent accretion, perhaps coming in with significant angular momentum, but with the gas being illuminated and photoionized by a powerful source of ionizing photons, i.e., highly obscured AGN and star formation that is being fueled by the same accretion event. In PRG1, this scenario would lead to the observed strong, spatially extended Ly $\alpha$, HeII, and metal line emission, and to the small velocity offset - primarily to the red - that is measured for Ly $\alpha$ This scenario would also be consistent with the observation of several Ly $\alpha$ nebulae that appear to be aligned with the filament of galaxies they reside in (Erb et al. 2011). One can imagine that the alignment is due to the preferential flow of material within the filament, perhaps entering a messy, rotating disk as it feeds a growing galaxy or protocluster. Detailed kinematic studies using high spatial resolution IFU observations to look for evidence of rotation or coherent flows within a larger number of Ly $\alpha$ nebulae would be ideal for testing this hypothesis.

\section{CONCLUSIONS}

Using the spatially extended emission in Ly $\alpha$ as well as in less optically thick emission lines, we study the role of scattering and the kinematics of the extended gas within PRG1, a Ly $\alpha$ nebula at $z \approx 1.67$. The low measured kinematic offset of $\operatorname{Ly} \alpha$ and the similarity of the surface brightness profiles observed in different emission lines are strong arguments that the extended Ly $\alpha$ is being produced in situ within the spatially extended gas, most likely due to photoionization from an AGN or distributed star formation, rather than scattered from a central source. The large-scale coherent velocity shear we observe across the entire nebula $-\approx 500 \mathrm{~km} \mathrm{~s}^{-1}$ over the central $\approx 50 \mathrm{kpc}$ - is broadly consistent with large-scale rotation in a clumpy, turbulent disk that is at least partially stable against collapse. Thus, while our data are inconsistent with cooling radiation powering the Ly $\alpha$ emission, accreting gas with high angular momentum flowing in along cold streams may explain the large scale coherent velocity structure that we observed within the extended Ly $\alpha$ nebula. This work suggests that, in at least some cases, the resonant Ly $\alpha$ line can be a robust tracer of the large-scale kinematics, and it motivates further deep spectroscopic studies of the extended gas within Ly $\alpha$ nebulae as a probe of the kinematics of the gas reservoir fueling episodes of active galaxy formation.

The authors thank Mark Dijkstra, Kristian Finlator, Peter Laursen, Norm Murray, and Anna Pancoast for illuminating discussions, Kristian Finlator for observing assistance, Alice Shapley for providing a comparison sky spectrum used to check the accuracy of our wavelength calibration, and the anonymous referee for useful suggestions that improved the quality of this paper. M.K.M.P. was supported by a TABASGO Prize Postdoctoral Fellowship and a Dark Cosmology Centre Postdoctoral Fellowship. This research was also supported in part by the National Science Foundation under AST1109288 (C.L.M.), and by NOAO (A.D.). NOAO is operated by the Association of Universities for Research in Astronomy (AURA), Inc. under a cooperative agreement with the National Science Foundation. AD's research is also partially supported by the Radcliffe Institute for Advanced Study and the Institute for Theory and Computation at Harvard University. AD thanks the Aspen Center for Physics, which is supported by the National Science Foundation Grant No. PHY-1066293.

The data presented herein were obtained at the W.M. Keck Observatory, which is operated as a scientific partnership among the California Institute of Technology, the University of California, and the National Aeronautics and Space Administration. The Observatory was made possible by the generous financial support of the W.M. Keck Foundation. The authors wish to recognize and acknowledge the very significant cultural role and reverence that the summit of Mauna Kea has always had within the indigenous Hawaiian community. We are most fortunate to have the opportunity to conduct observations from this mountain.

\section{REFERENCES}

8 In the context of AGN fluorescence, the presence of both blueshifted and redshift Ly $\alpha$ is easily understood, since the Ly $\alpha$ line is expected to exhibit either a prominent red or blue peak depending on the geometric alignment of the AGN with respect to the gas velocity field (Cantalupo et al. 2005).
Basu-Zych, A., \& Scharf, C. 2004, The Astrophysical Journal, $615, \mathrm{~L} 85$

Cantalupo, S., Porciani, C., Lilly, S. J., \& Miniati, F. 2005, The Astrophysical Journal, 628, 61

Cen, R., \& Zheng, Z. 2013, The Astrophysical Journal, 775, 112 
Chapman, S. C., Lewis, G. F., Scott, D., Richards, E., Borys, C., Steidel, C. C., Adelberger, K. L., \& Shapley, A. E. 2001, The Astrophysical Journal, 548, L17

Chapman, S. C., Scott, D., Windhorst, R. A., Frayer, D. T., Borys, C., Lewis, G. F., \& Ivison, R. J. 2004, The Astrophysical Journal, 606, 85

Colbert, J. W., Scarlata, C., Teplitz, H., Francis, P., Palunas, P., Williger, G. M., \& Woodgate, B. 2011, The Astrophysical Journal, 728,59

Dey, A., et al. 2005, The Astrophysical Journal, 629, 654

Dijkstra, M., Haiman, Z., \& Spaans, M. 2006, The Astrophysical Journal, 649, 14

Dijkstra, M., \& Loeb, A. 2009, Monthly Notices of the Royal Astronomical Society, 400, 1109

Duval, F., Schaerer, D., Östlin, G., \& Laursen, P. 2014, Astronomy \& Astrophysics, 562, A52

Erb, D. K., Bogosavljević, M., \& Steidel, C. C. 2011, The Astrophysical Journal, 740, L31

Fardal, M. A., Katz, N., Gardner, J. P., Hernquist, L., Weinberg, D. H., \& Dave, R. 2001, The Astrophysical Journal, 562, 605

Faucher-Giguère, C.-A., Kereš, D., Dijkstra, M., Hernquist, L., \& Zaldarriaga, M. 2010, The Astrophysical Journal, 725, 633

Förster Schreiber, N. M., et al. 2009, The Astrophysical Journal, 706, 1364

Francis, P. J., et al. 1996, The Astrophysical Journal, 457, 490

Geach, J. E., et al. 2009, The Astrophysical Journal, 700, 1

Genzel, R., et al. 2014, The Astrophysical Journal, 785, 75

Goerdt, T., Dekel, A., Sternberg, A., Ceverino, D., Teyssier, R., \& Primack, J. R. 2010, Monthly Notices of the Royal Astronomical Society, 407, 613

Guaita, L., Francke, H., Gawiser, E., Bauer, F. E., Hayes, M., Östlin, G., \& Padilla, N. 2013, Astronomy \& Astrophysics, 551, A93

Haiman, Z., Spaans, M., \& Quataert, E. 2000, The Astrophysical Journal, 537, L5

Hansen, M., \& Oh, S. P. 2006, Monthly Notices of the Royal Astronomical Society, 367, 979

Hashimoto, T., Ouchi, M., Shimasaku, K., Ono, Y., Nakajima, K., Rauch, M., \& Okamura, S. 2013, The Astrophysical Journal, 765, 70

Hayes, M., Scarlata, C., \& Siana, B. 2011, Nature, 476, 304

Kollmeier, J. A., Zheng, Z., Davé, R., Gould, A., Katz, N., Miralda-Escudé, J., \& Weinberg, D. H. 2010, The Astrophysical Journal, 708, 1048

Laursen, P., Duval, F., \& Östlin, G. 2013, The Astrophysical Journal, 766, 124

Laursen, P., Razoumov, A. O., \& Sommer-Larsen, J. 2009, The Astrophysical Journal, 696, 853

Madau, P. 1995, The Astrophysical Journal, 441, 18

Martin, D. C., Chang, D., Matuszewski, M., Morrissey, P., Rahman, S., Moore, A., Steidel, C. C., \& Matsuda, Y. 2014, 72

Massey, P., \& Gronwall, C. 1990, The Astrophysical Journal, 358, 344

Matsuda, Y., et al. 2004, The Astronomical Journal, 128, 569

Matsuda, Y., et al. 2005, The Astrophysical Journal, 634, L125

Matsuda, Y., et al. 2009, Monthly Notices of the Royal Astronomical Society: Letters, 400, L66

Matsuda, Y., et al. 2011, Monthly Notices of the Royal Astronomical Society: Letters, 410, L13

McLinden, E. M., Malhotra, S., Rhoads, J. E., Hibon, P., Weijmans, A.-M., \& Tilvi, V. 2013, arXiv preprint arXiv: ..., 1

McLinden, E. M., et al. 2011, The Astrophysical Journal, 730, 136

Mori, M., Umemura, M., \& Ferrara, A. 2004, The Astrophysical Journal, 613, L97

Neistein, E., van den Bosch, F. C., \& Dekel, A. 2006, Monthly Notices of the Royal Astronomical Society, 372, 933

Neufeld, D. A. 1990, The Astrophysical Journal, 350, 216

Newman, S. F., et al. 2012, The Astrophysical Journal, 761, 43

Oke, J. B. 1974, The Astrophysical Journal Supplement Series, 27,21
Oke, J. B., et al. 1995, Publications of the Astronomical Society of the Pacific, 107, 375

Osterbrock, D. E. 1989, Astrophysics of gaseous nebulae and active galactic nuclei, ed. Osterbrock, D. ${ }^{2} \mathrm{E}$.

Overzier, R. A., Nesvadba, N. P. H., Dijkstra, M., Hatch, N. A., Lehnert, M. D., Villar-Martín, M., Wilman, R. J., \& Zirm, A. W. 2013, 12

Peterson, B. M. 1997, An introduction to active galactic nuclei

Prescott, M. K. M. 2009, Phd, University of Arizona

Prescott, M. K. M., Dey, A., \& Jannuzi, B. T. 2009, The Astrophysical Journal, 702, 554

Prescott, M. K. M., Dey, A., \& Jannuzi, B. T. 2012a, The Astrophysical Journal, 748, 125

Prescott, M. K. M., Dey, A., \& Jannuzi, B. T. 2013, The Astrophysical Journal, 762, 38

Prescott, M. K. M., Kashikawa, N., Dey, A., \& Matsuda, Y. 2008, The Astrophysical Journal, 678, L77

Prescott, M. K. M., Smith, P. S., Schmidt, G. D., \& Dey, A. 2011, The Astrophysical Journal, 730, L25

Prescott, M. K. M., et al. 2012b, The Astrophysical Journal, 752, 86

Rosdahl, J., \& Blaizot, J. 2012, Monthly Notices of the Royal Astronomical Society, 423, 344

Saito, T., Shimasaku, K., Okamura, S., Ouchi, M., Akiyama, M., \& Yoshida, M. 2006, The Astrophysical Journal, 648, 54

Song, M., et al. 2014, eprint arXiv:1406.4503

Steidel, C. C., Adelberger, K. L., Shapley, A. E., Pettini, M. Dickinson, M., \& Giavalisco, M. 2000, The Astrophysical Journal, 532, 170

Steidel, C. C., Bogosavljević, M., Shapley, A. E., Kollmeier, J. A., Reddy, N. A., Erb, D. K., \& Pettini, M. 2011, The Astrophysical Journal, 736, 20

Steidel, C. C., Erb, D. K., Shapley, A. E., Pettini, M., Reddy, N., Bogosavljević, M., Rudie, G. C., \& Rakic, O. 2010, The Astrophysical Journal, 717, 289

Stewart, K. R., Brooks, A. M., Bullock, J. S., Maller, A. H., Diemand, J., Wadsley, J., \& Moustakas, L. A. 2013, The Astrophysical Journal, 769, 74

Stewart, K. R., Kaufmann, T., Bullock, J. S., Barton, E. J., Maller, A. H., Diemand, J., \& Wadsley, J. 2011, The Astrophysical Journal, 738, 39

Storey, P. J., \& Hummer, D. G. 1995, Monthly Notices of the Royal Astronomical Society, 272, 41

Taniguchi, Y., \& Shioya, Y. 2000, The Astrophysical Journal, 532, L13

Taniguchi, Y., Shioya, Y., \& Kakazu, Y. 2001, The Astrophysical Journal, 562, L15

Verhamme, A., Schaerer, D., \& Maselli, A. 2006, Astronomy and Astrophysics, 460, 397

Verner, D. A., Ferland, G. J., Korista, K. T., \& Yakovlev, D. G. 1996, The Astrophysical Journal, 465, 487

Webb, T. M. A., Yamada, T., Huang, J.-S., Ashby, M. L. N., Matsuda, Y., Egami, E., Gonzalez, M., \& Hayashimo, T. 2009, The Astrophysical Journal, 692, 1561

Yang, Y., Walter, F., Decarli, R., Bertoldi, F., Weiss, A., Dey, A., Prescott, M. K. M., \& Bdescu, T. 2014a, The Astrophysical Journal, 784, 171

Yang, Y., Zabludoff, A., Eisenstein, D., \& Davé, R. 2010, The Astrophysical Journal, 719, 1654

Yang, Y., Zabludoff, A., Jahnke, K., \& Davé, R. 2014b, The Astrophysical Journal, 793, 114

Yang, Y., Zabludoff, A., Jahnke, K., Eisenstein, D., Davé, R., Shectman, S. A., \& Kelson, D. D. 2011, The Astrophysical Journal, 735, 87

Yang, Y., Zabludoff, A., Tremonti, C., Eisenstein, D., \& Davé, R. 2009, The Astrophysical Journal, 693, 1579

Yang, Y., Zabludoff, A. I., Dave, R., Eisenstein, D. J., Pinto, P. A., Katz, N., Weinberg, D. H., \& Barton, E. J. 2006, The Astrophysical Journal, 640, 539

Yang, Y., et al. 2012, The Astrophysical Journal, 744, 178

Zheng, Z., Cen, R., Weinberg, D., Trac, H., \& Miralda-Escudé, J. 2011, The Astrophysical Journal, 739, 62 
TABLE 1

OBSERVING LoG

\begin{tabular}{|c|c|c|c|c|c|c|c|c|c|}
\hline UT Date & $\begin{array}{l}\text { UT Time } \\
\text { Range }\end{array}$ & $\begin{array}{c}\text { Parallactic } \\
\text { Angle Range }\end{array}$ & $\begin{array}{l}\text { Right Ascension }{ }^{\mathrm{a}} \\
\text { (hours) }\end{array}$ & $\begin{array}{l}\text { Declination }^{\mathrm{a}} \\
\text { (degrees) }\end{array}$ & $\begin{array}{l}\text { Position } \\
\text { Angle }\end{array}$ & $\begin{array}{l}\text { Exposure }^{\mathrm{b}} \\
\text { Time (sec) }\end{array}$ & $\begin{array}{l}\text { Slitwidth } \\
\text { (arcsec) }\end{array}$ & $\begin{array}{l}\text { Seeing } \\
(\operatorname{arcsec})\end{array}$ & Conditions \\
\hline 2009 April 25 & $10: 00-12: 45$ & $-145^{\circ}-111^{\circ}$ & $14: 35: 12.385$ & $35: 11: 06.62$ & $52.44^{\circ}$ & $8300 / 8100$ & 1.2 & $0.8-1.0$ & Clear \\
\hline 2009 April 25 & $\begin{array}{l}8: 00-10: 00 \\
13: 00-14: 30\end{array}$ & $\begin{array}{l}78^{\circ}-35^{\circ} \\
108^{\circ}-91^{\circ}\end{array}$ & $14: 35: 12.385$ & $35: 11: 06.62$ & $146.0^{\circ}$ & 7630 / 7200 & 1.2 & $1.0-1.1$ & Clear \\
\hline 2010 May 10 & $6: 45-10: 00$ & $-99^{\circ}-167^{\circ}$ & $14: 35: 12.385$ & $35: 11: 06.62$ & $52.44^{\circ}$ & $10350 / 9000$ & 1.5 & $0.8-0.9$ & Clear \\
\hline 2010 May 10 & $10: 45-14: 15$ & $135^{\circ}-85^{\circ}$ & $14: 35: 12.385$ & $35: 11: 06.62$ & $146.0^{\circ}$ & 10350 / 9900 & 1.5 & $0.7-0.8$ & Clear \\
\hline
\end{tabular}

${ }^{a}$ Location on the nebula where the two slits cross.

b Values listed separately for LRIS-Blue / LRIS-Red. 
TABLE 2

Spectroscopic Calibration

\begin{tabular}{|c|c|c|c|c|c|c|c|}
\hline UT Date & $\begin{array}{l}\text { Position } \\
\text { Angle }\end{array}$ & Detector & $\begin{array}{l}\text { Dispersion } \\
(\AA / \text { pix })\end{array}$ & $\begin{array}{l}\text { Resolution FWHM } \\
\qquad\left(\mathrm{km} \mathrm{s}^{-1}\right)\end{array}$ & $\begin{array}{l}\text { Wavelength } \\
\text { Range }(\AA)\end{array}$ & $\begin{array}{c}\text { Wavelength Solution } \\
\operatorname{rms}(\AA)\end{array}$ & $\begin{array}{c}\text { Sky Line Comparison }{ }^{\mathrm{a}} \\
\text { rms }(\AA)\end{array}$ \\
\hline \multirow[t]{2}{*}{2009 April 25} & $52.44^{\circ}$ & LRIS-Blue & 1.09 & $758-501$ & $3100-5600$ & 0.24 & 0.32 \\
\hline & & LRIS-Red & 1.86 & $471-350$ & $5600-10000$ & 0.11 & 0.20 \\
\hline \multirow[t]{2}{*}{2009 April 25} & $146.0^{\circ}$ & LRIS-Blue & 1.09 & $758-501$ & $3100-5600$ & 0.24 & 0.25 \\
\hline & & LRIS-Red & 1.86 & $471-350$ & $5600-10000$ & 0.12 & 0.42 \\
\hline \multirow[t]{2}{*}{2010 May 10} & $52.44^{\circ}$ & LRIS-Blue & 2.18 & $966-554$ & $3100-6800$ & 0.14 & 0.14 \\
\hline & & LRIS-Red & 1.16 & $439-292$ & $6800-10870$ & 0.21 & 0.25 \\
\hline \multirow[t]{2}{*}{2010 May 10} & $146.0^{\circ}$ & LRIS-Blue & 2.18 & $966-554$ & $3100-6800$ & 0.16 & 0.17 \\
\hline & & LRIS-Red & 1.16 & $439-292$ & $6800-10870$ & 0.28 & 0.42 \\
\hline
\end{tabular}

a Measured rms relative to sky lines HgI $\lambda 4047, \mathrm{HgI} \lambda 5461$ (2009), [O I] $\lambda 5577$, and [OI] $\lambda 6300$ (2010) for LRIS-Blue and [O I] $\lambda 6364$, OH8-3(P1,2) $\lambda 7316$,

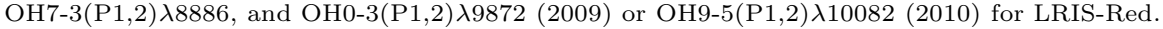

TABLE 3

Surface Brightness Profile Sizes

\begin{tabular}{|c|c|c|c|c|c|c|c|}
\hline PA & Line & $\begin{array}{c}\mathrm{D}_{50}{ }^{\mathrm{a}} \\
(\operatorname{arcsec})\end{array}$ & $\begin{array}{l}\mathrm{D}_{50} \\
(\mathrm{kpc})\end{array}$ & $\begin{array}{c}\mathrm{F}_{50}{ }^{\mathrm{b}} \\
\left(10^{-17} \mathrm{erg} \mathrm{s}^{-1} \mathrm{~cm}^{-2}\right)\end{array}$ & $\begin{array}{c}\mathrm{D}_{90}{ }^{\mathrm{a}} \\
(\operatorname{arcsec})\end{array}$ & $\begin{array}{c}\mathrm{D}_{90} \\
(\mathrm{kpc})\end{array}$ & $\begin{array}{c}\mathrm{F}_{90} \mathrm{~b} \\
\left(10^{-17} \mathrm{erg} \mathrm{s}^{-1} \mathrm{~cm}^{-2}\right)\end{array}$ \\
\hline $52^{\circ} .44$ & $\begin{array}{c}\text { Ly } \alpha \\
\text { HeII } \\
\text { CIV+HeII+CIII }]\end{array}$ & $\begin{array}{l}3.05 \pm 0.11 \\
2.29 \pm 0.16 \\
2.36 \pm 0.13\end{array}$ & $\begin{array}{l}25.87 \pm 0.94 \\
19.43 \pm 1.38 \\
19.96 \pm 1.09\end{array}$ & $\begin{array}{c}60.5 \pm 2.0 \\
5.1 \pm 0.3 \\
11.3 \pm 0.6\end{array}$ & $\begin{array}{l}7.14 \pm 0.37 \\
6.34 \pm 0.84 \\
5.64 \pm 0.67\end{array}$ & $\begin{array}{l}60.45 \pm 3.14 \\
53.67 \pm 7.11 \\
47.73 \pm 5.64\end{array}$ & $\begin{array}{c}108.9 \pm 3.0 \\
9.1 \pm 0.5 \\
20.3 \pm 1.0\end{array}$ \\
\hline 146.0 & $\begin{array}{c}\text { Ly } \alpha \\
\text { HeII } \\
\text { CIV+HeII+CIII }]\end{array}$ & $\begin{array}{l}2.29 \pm 0.13 \\
1.91 \pm 0.17 \\
1.72 \pm 0.12\end{array}$ & $\begin{array}{l}19.39 \pm 1.08 \\
16.19 \pm 1.43 \\
14.54 \pm 1.01\end{array}$ & $\begin{array}{l}43.8 \pm 2.0 \\
3.9 \pm 0.3 \\
7.5 \pm 0.5\end{array}$ & $\begin{array}{l}7.00 \pm 0.60 \\
4.79 \pm 0.59 \\
4.25 \pm 0.38\end{array}$ & $\begin{array}{l}59.32 \pm 5.10 \\
40.53 \pm 5.02 \\
36.01 \pm 3.22\end{array}$ & $\begin{array}{c}79.1 \pm 3.4 \\
7.0 \pm 0.5 \\
13.5 \pm 0.8\end{array}$ \\
\hline
\end{tabular}

${ }^{a}$ Diameter containing $50 \%$ or $90 \%$ of the total flux measured in a given emission line.

b Total flux contained within $\mathrm{D}_{50}$ or $\mathrm{D}_{90}$ in a given emission line. 
TABLE 4

Toy Model Fit Parameters

\begin{tabular}{cc}
\hline \hline Parameter & $\begin{array}{c}67 \% \text { Confidence } \\
\text { Interval }\end{array}$ \\
\hline$\Theta_{\text {off }}$ & {$\left[-30^{\circ},-16^{\circ}\right]$} \\
$i$ & {$\left[38^{\circ}, 77^{\circ}\right]$} \\
$R_{\max }$ & {$\left[0.8^{\prime \prime}, 2.3^{\prime \prime}\right]$} \\
$V_{\max }$ & {$[233,441]$} \\
$X_{c}$ & {$\left[-0.9^{\prime \prime},-0.3^{\prime \prime}\right]$} \\
$Y_{c}$ & {$\left[-0.7^{\prime \prime},-0.4^{\prime \prime}\right]$}
\end{tabular}
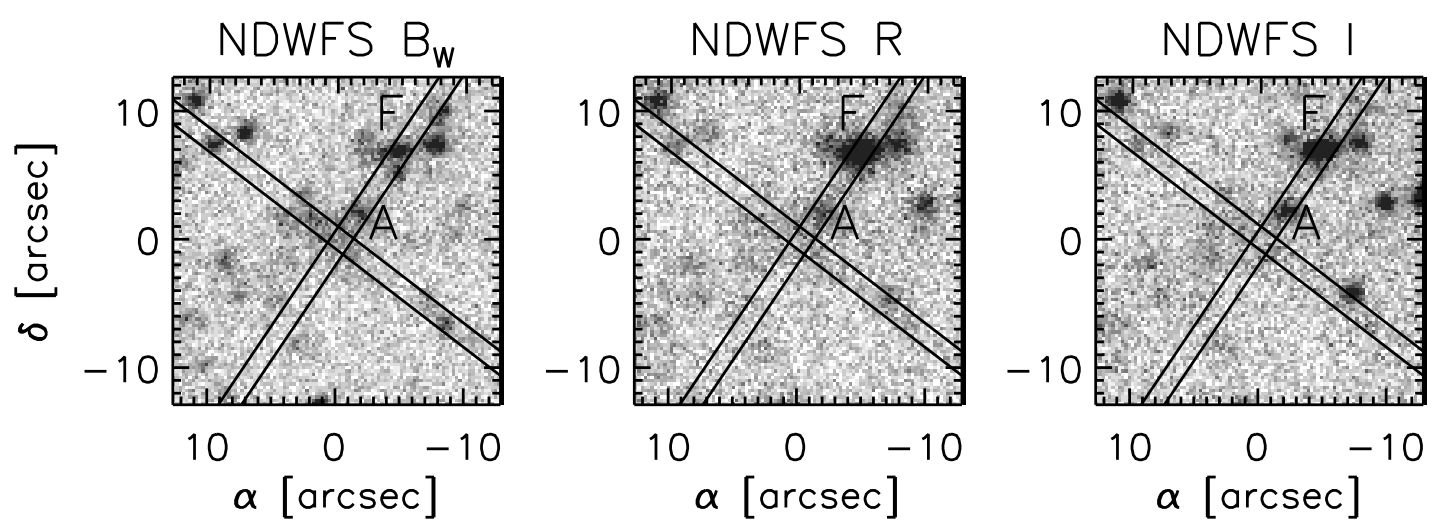

FIG. 1. - Broad-band $B_{W}, R$, and $I$-band imaging of PRG1, oriented with $\mathrm{N}$ up and $\mathrm{E}$ to the left, overlaid with the two slit positions used for spectroscopic observations $\left(\mathrm{PA}=52^{\circ} .44\right.$ and $\left.\mathrm{PA}=146^{\circ} 0\right)$. Diffuse continuum emission from the nebula is visible, particularly in the $B_{W}$ image (Prescott et al. 2013). In each panel, the origin is located at 14:35:12.385 +35:11:06.62, the location where the two slits cross, and Source 'A' - the red, compact source at the northwest edge of the nebula - is labeled. Note that the source labeled ' $F$,' the bright galaxy intersecting the $\mathrm{PA}=146^{\circ} 0$ slit at $\left[-5^{\prime \prime}, 7^{\prime \prime}\right]$, is a $z \approx 0.479$ object, with unambiguous $[\mathrm{OII}], \mathrm{H} \beta$, and [OIII] emission visible in the LRIS spectroscopy. 

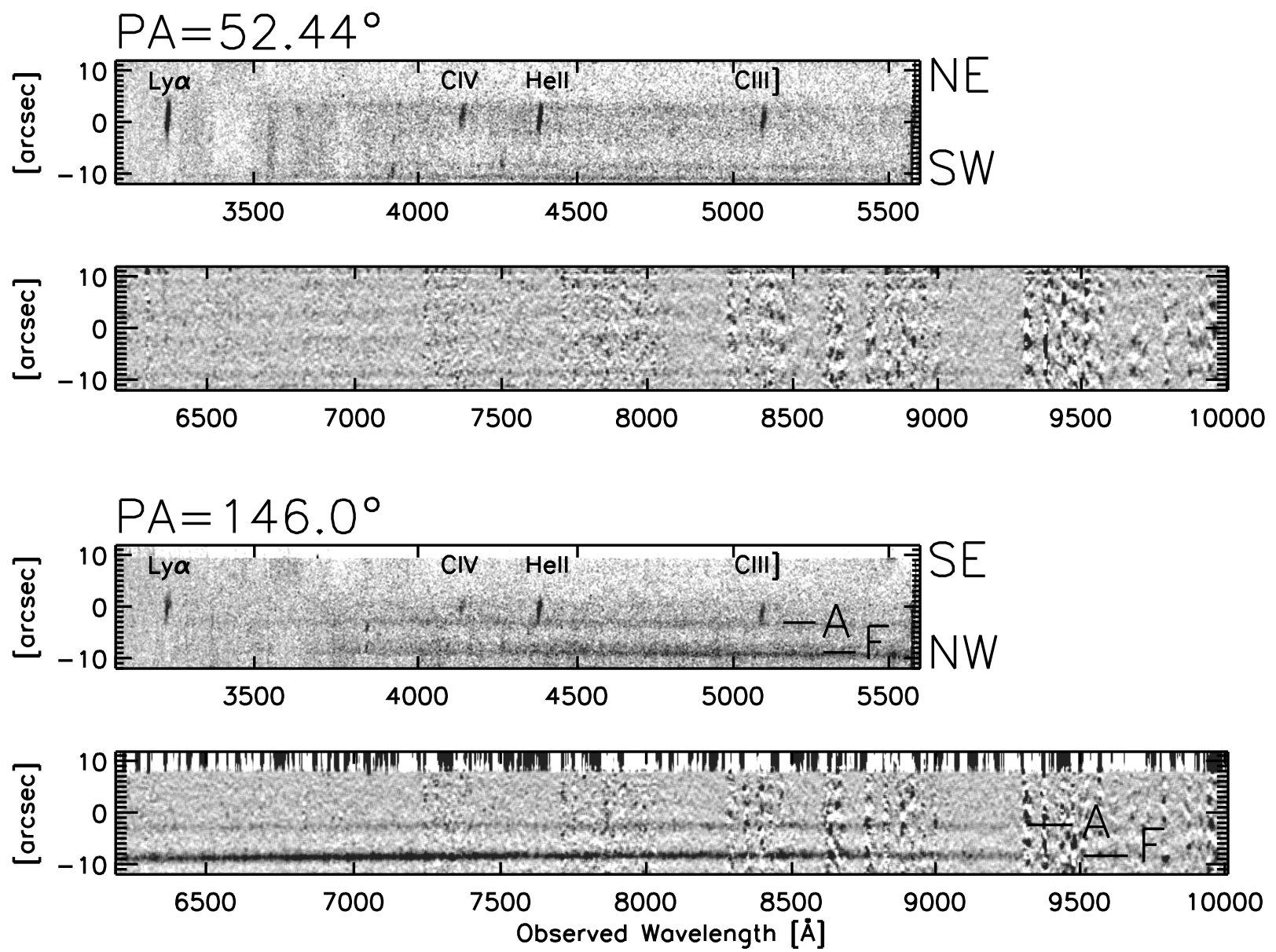

Fig. 2.- Individual $2 \mathrm{D}$ spectra at $\mathrm{PA}=52^{\circ} 44$ and $\mathrm{PA}=146.0$ from the 2009 run (binned by two in the spectral dimension, to match the 2010 data) prior to flux calibration. Emission lines are labeled along with the positions of Sources 'A' and ' $F$ ' on the slit. Zero in the spatial direction corresponds to the position where the two slits cross, as listed in Table 1 

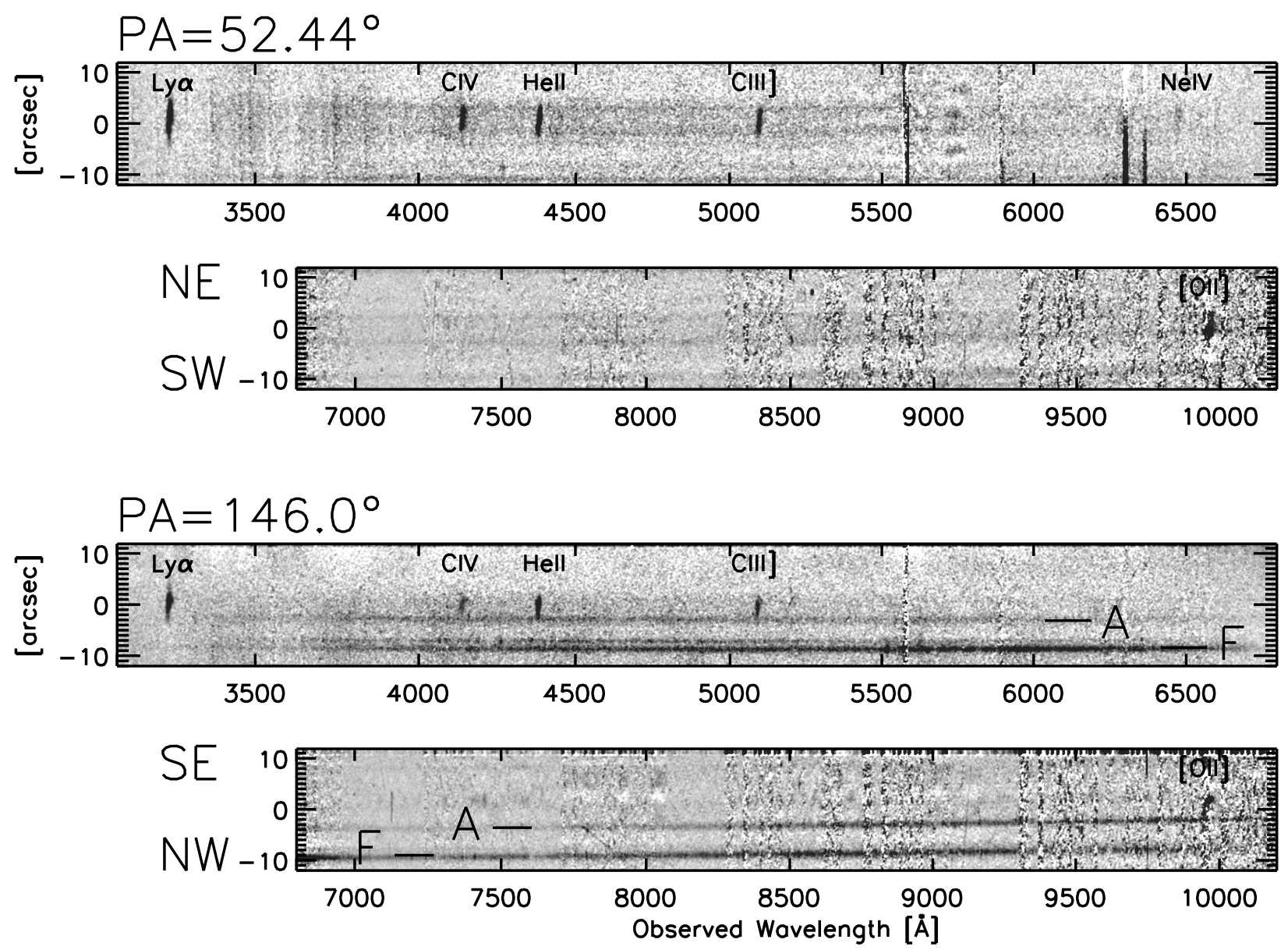

FIG. 3.- Individual 2D spectra at $\mathrm{PA}=52^{\circ} .44$ and $\mathrm{PA}=146^{\circ} 0$ from the 2010 run prior to flux calibration. Emission lines are labeled along with the positions of Sources 'A' and ' $\mathrm{F}$ ' on the slit. Zero in the spatial direction corresponds to the position where the two slits cross, as listed in Table 1. 

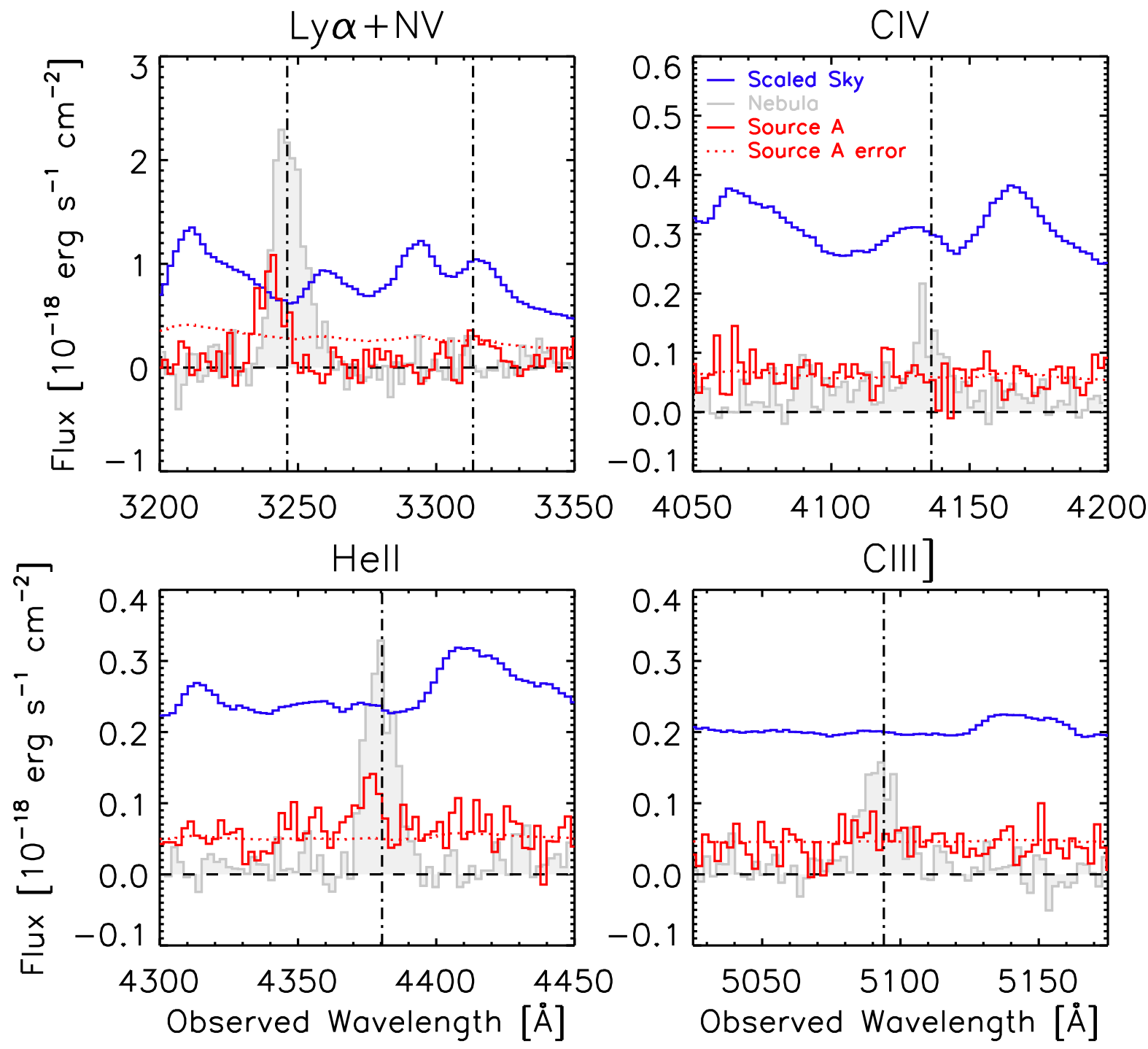

Fig. 4.- Source A spectrum at the locations of Ly $\alpha, \mathrm{NV}, \mathrm{CIV}$, HeII, and CIII] at the redshift of PRG1, extracted from a 5 pixel (0.67") aperture (red solid line), with the corresponding error spectrum overplotted (red dotted line). The spectrum taken from the center of the nebula is shown as the filled grey region, and a scaled sky spectrum is shown in blue. The spectrum of Source A shows strong Ly $\alpha$ emission and a tentative detection of HeII, most likely emission from the nebula overlapping the position of Source A. There is no independent evidence from the spectrum of Source A that confirms that it lies at the redshift of PRG1. However, based on its proximity and the lack of continuum emission at $\lambda_{\text {obs }}<3230 \AA$, it is plausible that this source is associated with PRG1. 


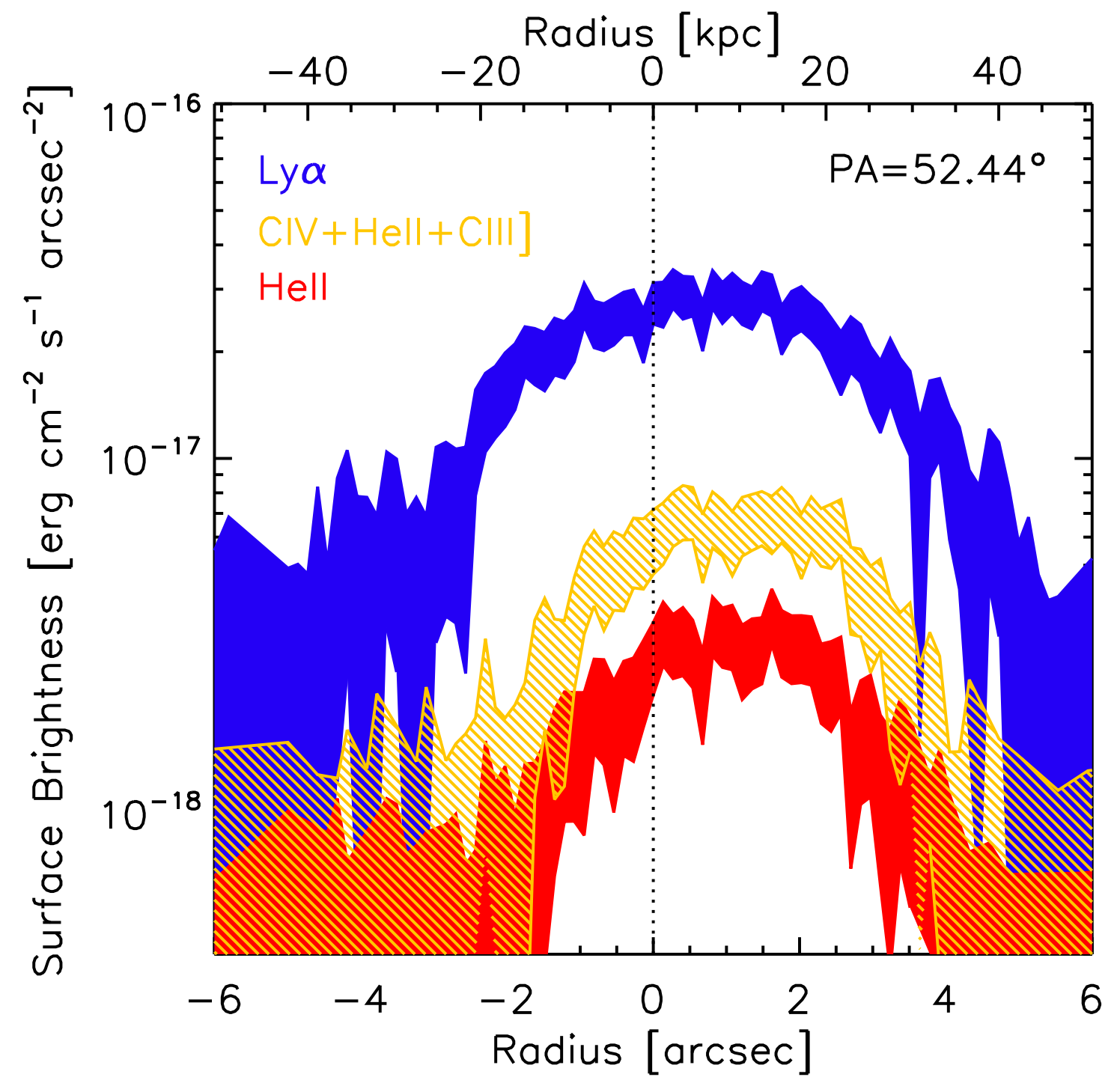

FIG. 5.- Surface brightness profiles of Ly $\alpha$ (solid blue region), HeII (solid red region), and a combined CIV+HeII + CIII] (hashed yellow region) along the $\mathrm{PA}=52^{\circ} .44$ slit. The filled regions span the range between the upper and lower error bars for each bin. The plot is centered in the spatial direction about the position where the two slits cross (dotted line). For clarity, we restrict the x-axis of the plot to the range over which we have good signal-to-noise ratio measurements. 


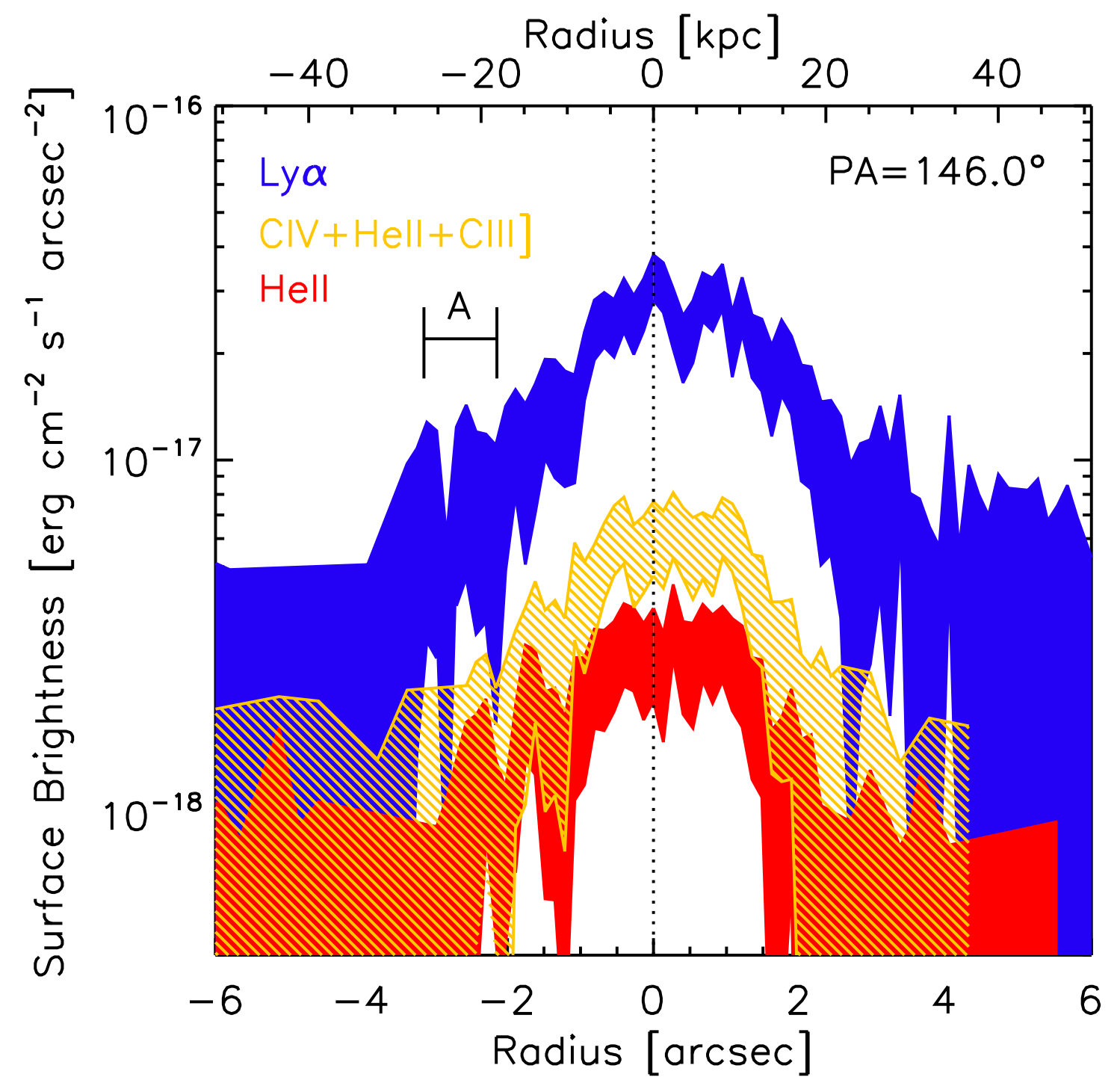

FIG. 6. - Surface brightness profiles of Ly $\alpha$ (solid blue region), HeII (solid red region), and a combined CIV+HeII + CIII] (hashed yellow region) along the $\mathrm{PA}=146^{\circ} 0$ slit. The filled regions span the range between the upper and lower error bars for each bin. The plot is centered in the spatial direction about the position where the two slits cross (dotted line). For clarity, we restrict the x-axis of the plot to the range over which we have good signal-to-noise ratio measurements. The location of Source A is indicated. 

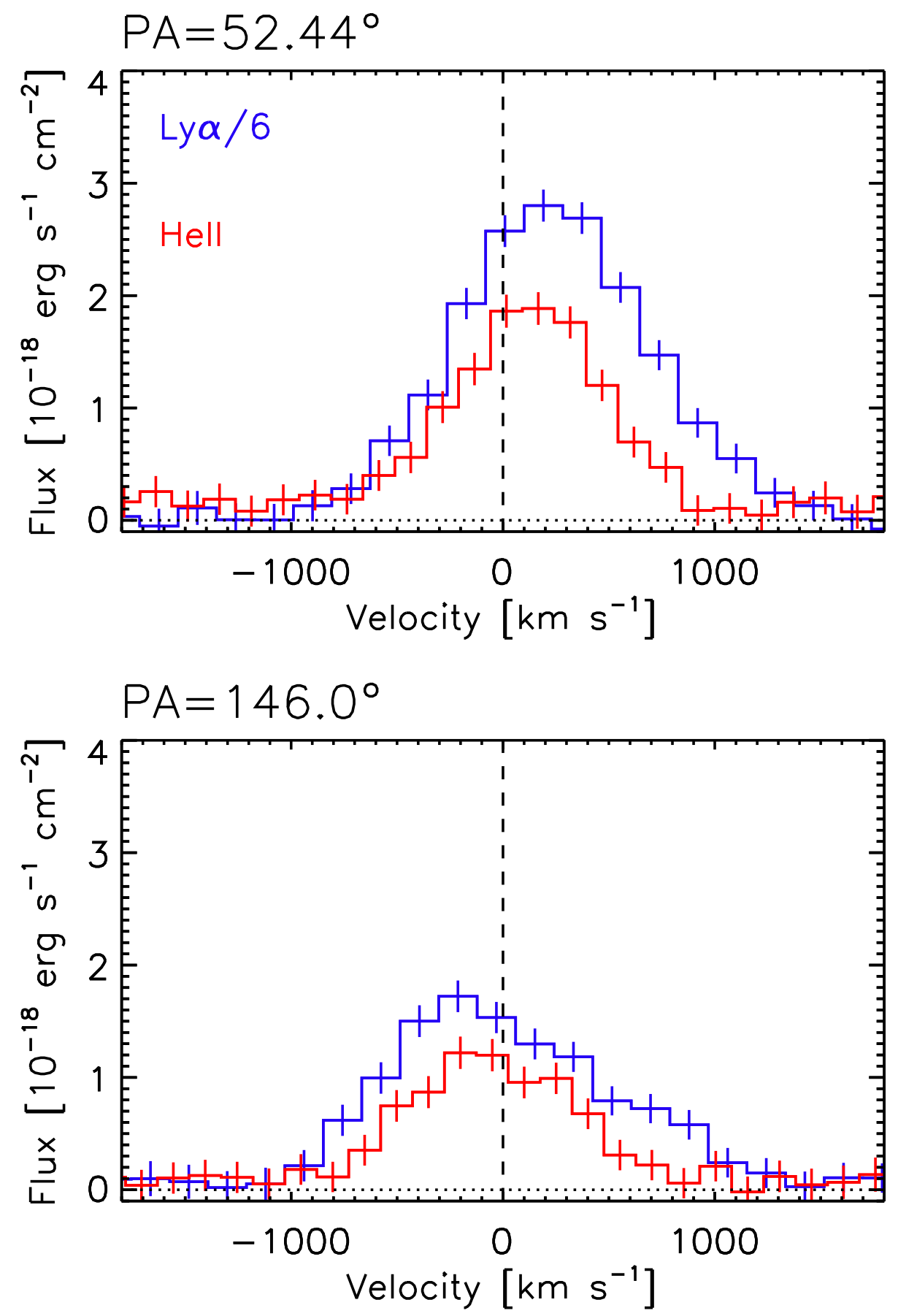

FIG. 7.- Emission line profiles of $\operatorname{Ly} \alpha$ (blue line, scaled down for clarity) and HeII (red line) measured within $7.2^{\prime \prime}$ and 5.8 $8^{\prime \prime}$ wide apertures, respectively, at $\mathrm{PA}=52^{\circ} .44$ and $\mathrm{PA}=146^{\circ} .0$. The vertical dashed line in each panel corresponds to the systemic velocity defined as the centroid of HeII at the position on the nebula where the two slits cross. A positive velocity corresponds to a redshift relative to the systemic velocity. 


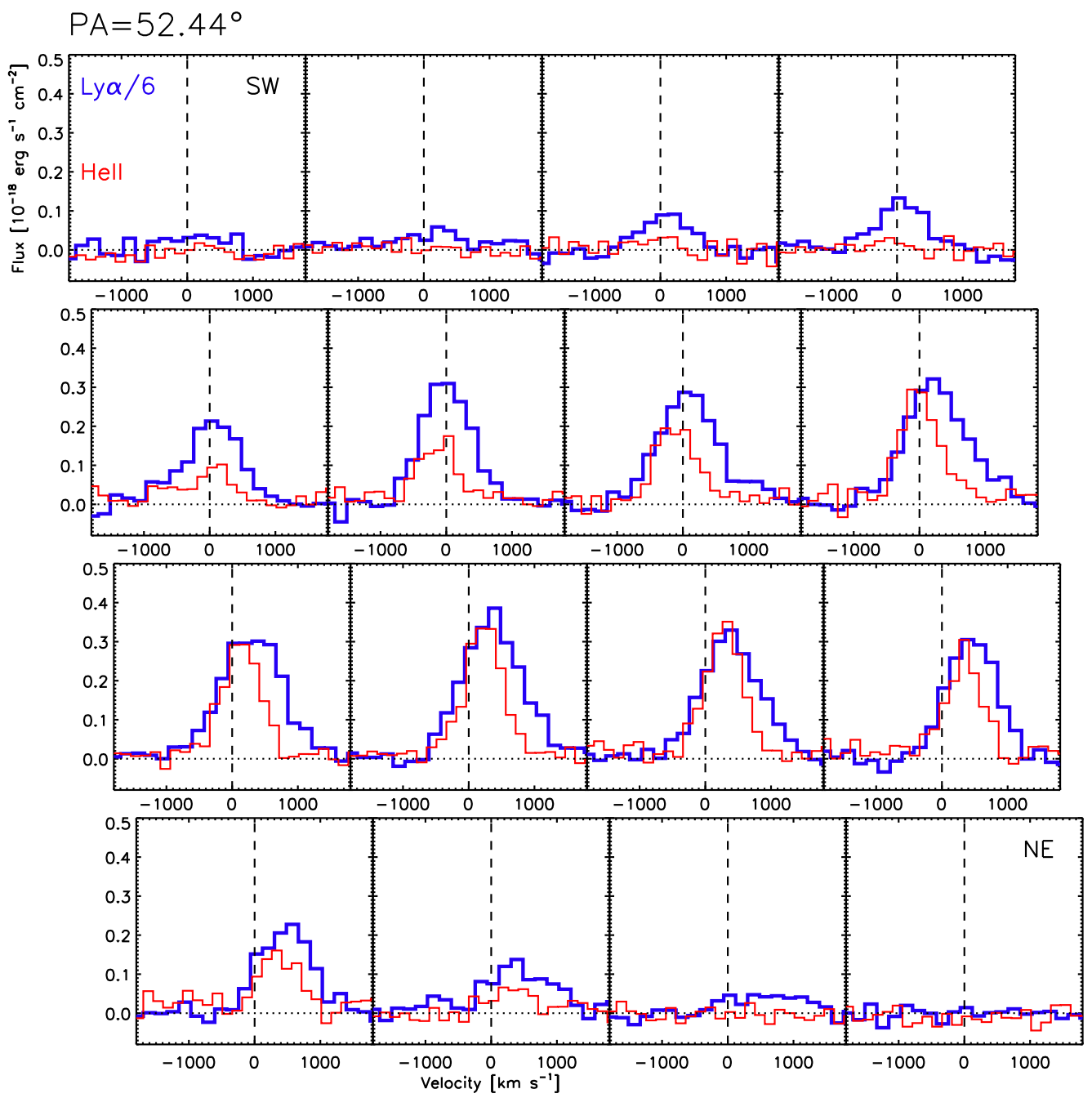

FIG. 8. - Emission line profiles of Ly $\alpha$ (blue line, scaled down for clarity) and HeII (red line) measured as a function of position from the SW (upper left) to the NE (lower right) end of the $\mathrm{PA}=52^{\circ} .44$ slit, extracted in 5 pix $=0.67^{\prime \prime}$ apertures. The vertical dashed line in each panel corresponds to the systemic velocity defined as the centroid of HeII at the position on the nebula where the two slits cross. A positive velocity corresponds to a redshift relative to the systemic velocity. 


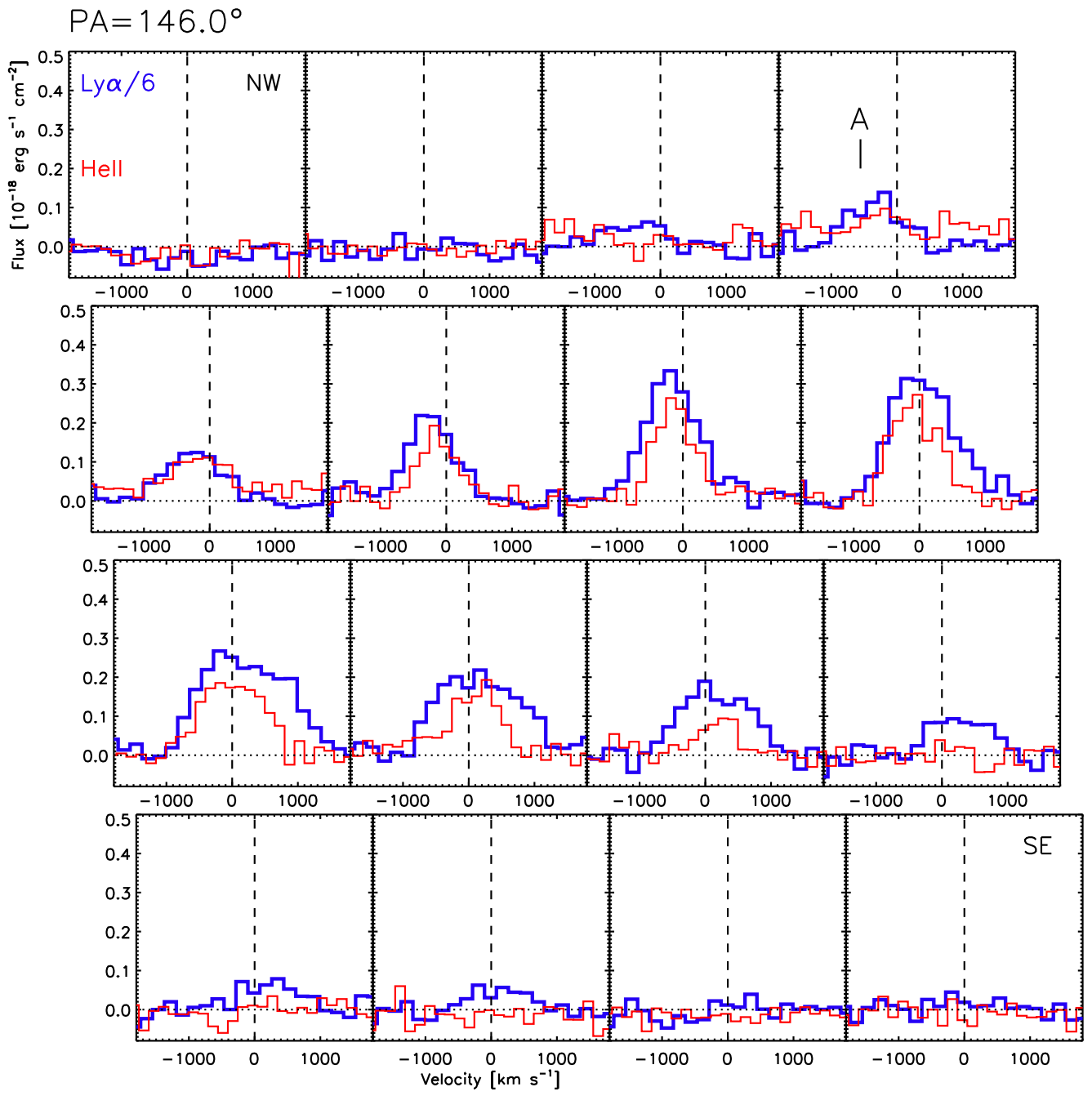

FIG. 9. - Emission line profiles of Ly $\alpha$ (blue line, scaled down for clarity) and HeII (red line) measured as a function of position from the NW (upper left) to the SE (lower right) end of the $\mathrm{PA}=146^{\circ} 0$ slit, extracted in 5 pix $=0.67^{\prime \prime}$ apertures. The vertical dashed line in each panel corresponds to the systemic velocity defined as the centroid of HeII at the position on the nebula where the two slits cross. A positive velocity corresponds to a redshift relative to the systemic velocity. The position of Source A is indicated. 


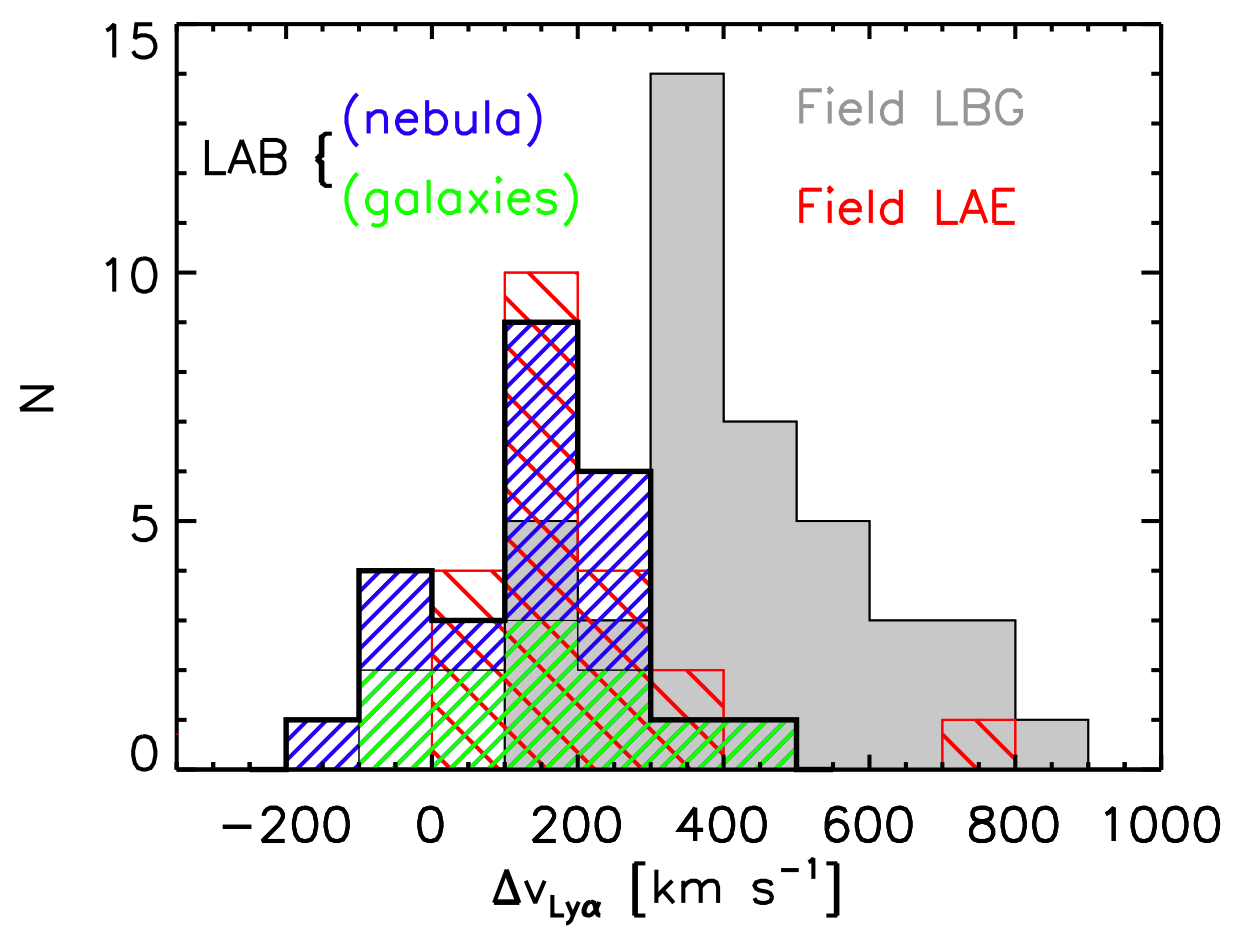

FIG. 10.- Velocity offset between Ly $\alpha$ and the systemic velocity for LBGs (filled grey histogram; Steidel et al. 2010) and LAEs (narrow hashed red histogram; McLinden et al. 2011; Guaita et al. 2013; Hashimoto et al.|2013; Song et al. 2014). Velocity offsets measured within LABs are shown, as measured at the position of embedded galaxies (slanted hashed green histogram; Francis et al. 1996; McLinden et al. 2013; Yang et al. 2014b) and from spatial apertures within the extended nebula (slanted hashed blue histogram; thirteen measurements are from this work on PRG1, measured within 5 pix $\approx 0.67^{\prime \prime}$ spatial apertures with a minimum of $S N R=3$ in both Ly $\alpha$ and HeII, and one additional measurement is taken from LABd05 by (Yang et al. 2014a). Ly $\alpha$ nebulae show Ly $\alpha$ velocity offsets that are lower than those measured for the bulk of the LBG population but similar or slightly lower than what is seen in LAEs. 

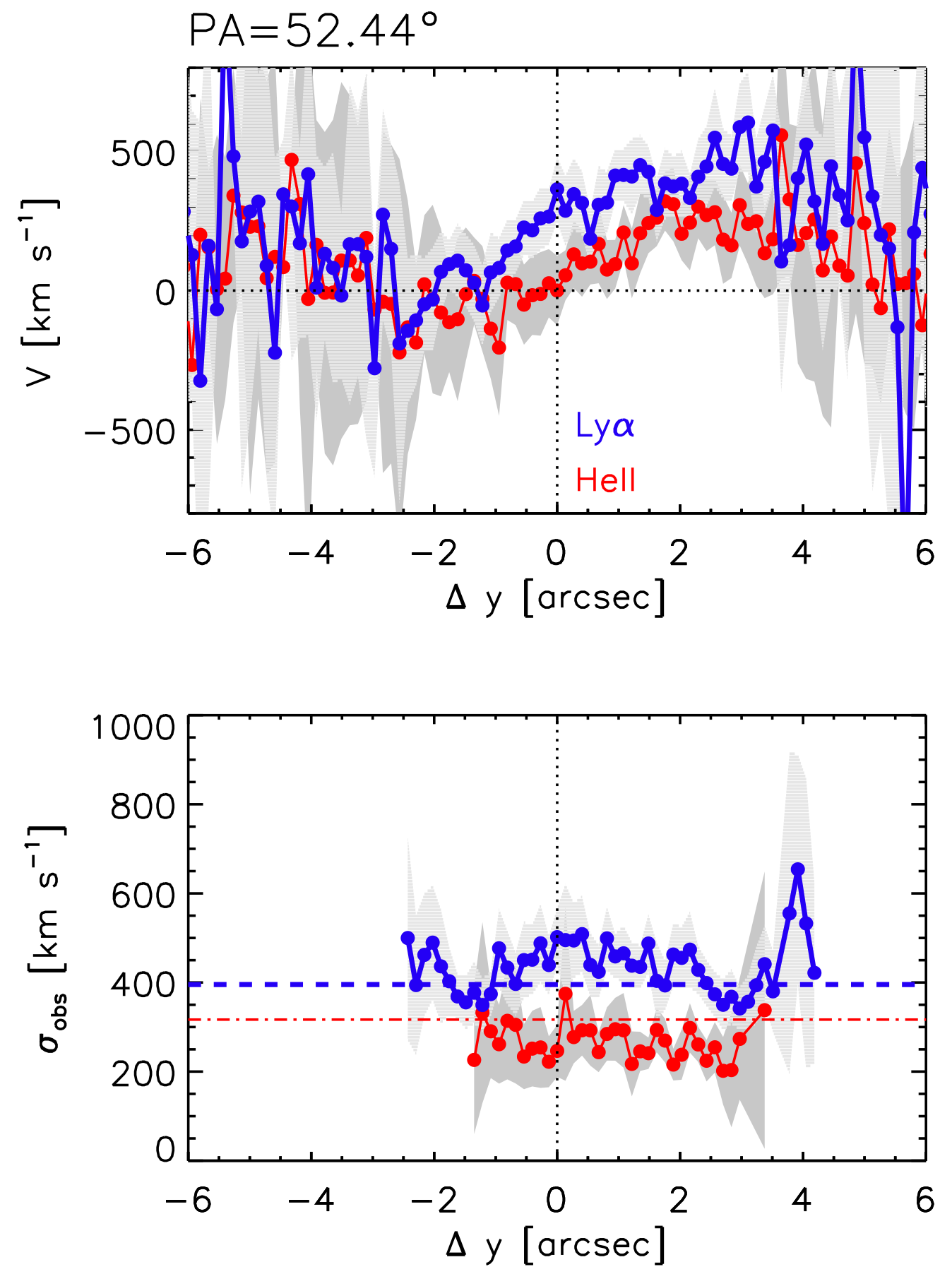

FIG. 11.- Rotation (top panel) and velocity dispersion (bottom panel) curves for Ly $\alpha$ and HeII for the PA $=52^{\circ} .44$. Ly $\alpha$ is shown as a thick blue line, and HeII is shown as a thin red line. The hashed grey and grey-shaded regions indicate the corresponding error bars. The velocity dispersion is the observed value, with the instrumental resolution at the location of Ly $\alpha$ and HeII shown as blue dashed and red dot-dashed lines, respectively. 

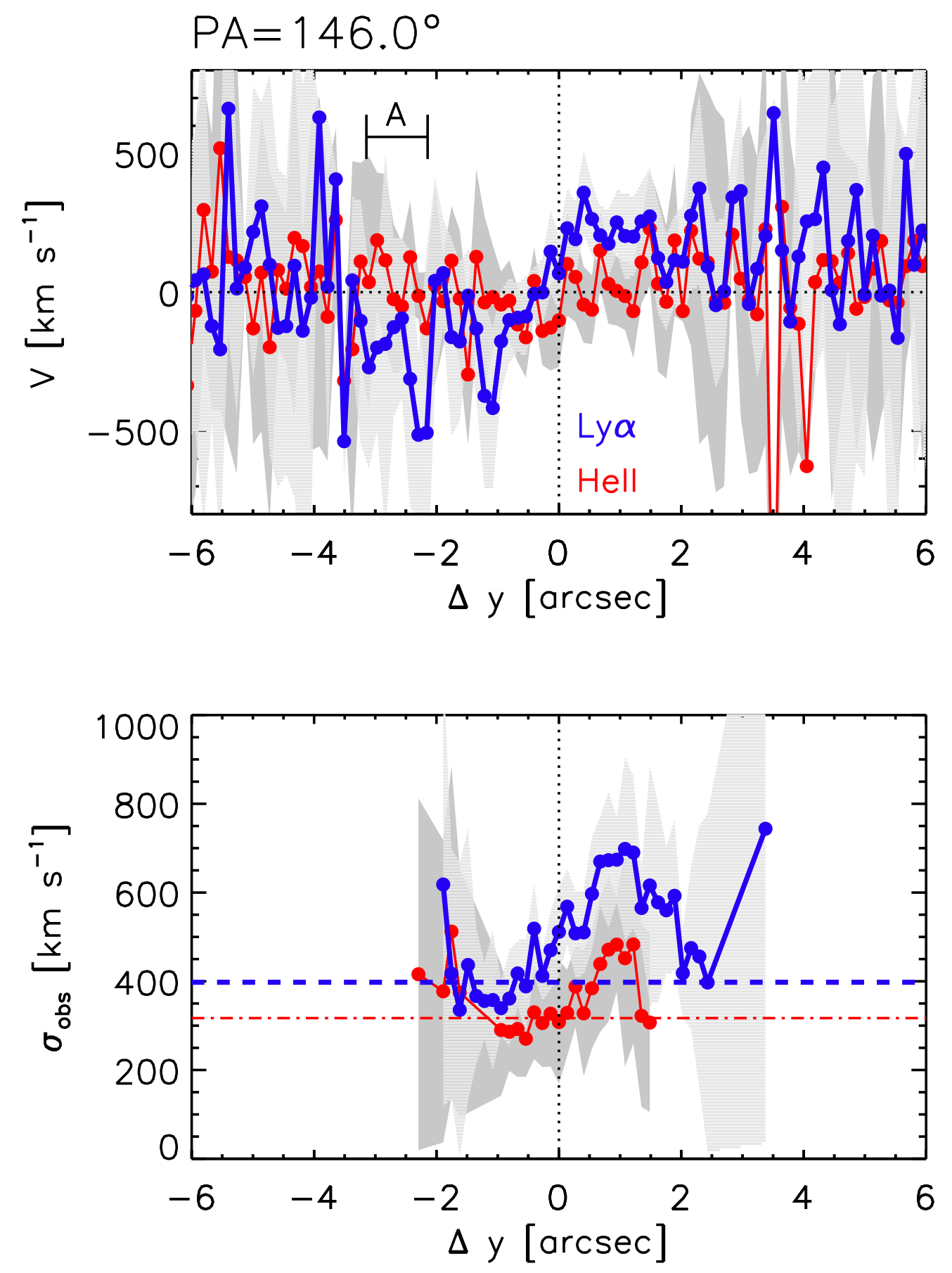

FIG. 12.- Rotation (top panel) and velocity dispersion (bottom panel) curves for Ly $\alpha$ and HeII for the PA=146.0. Ly $\alpha$ is shown as a thick blue line, and HeII is shown as a thin red line. The hashed grey and grey-shaded regions indicate the corresponding error bars. The velocity dispersion is the observed value, with the instrumental resolution at the location of Ly $\alpha$ and HeII shown as blue dashed and red dot-dashed lines, respectively. 

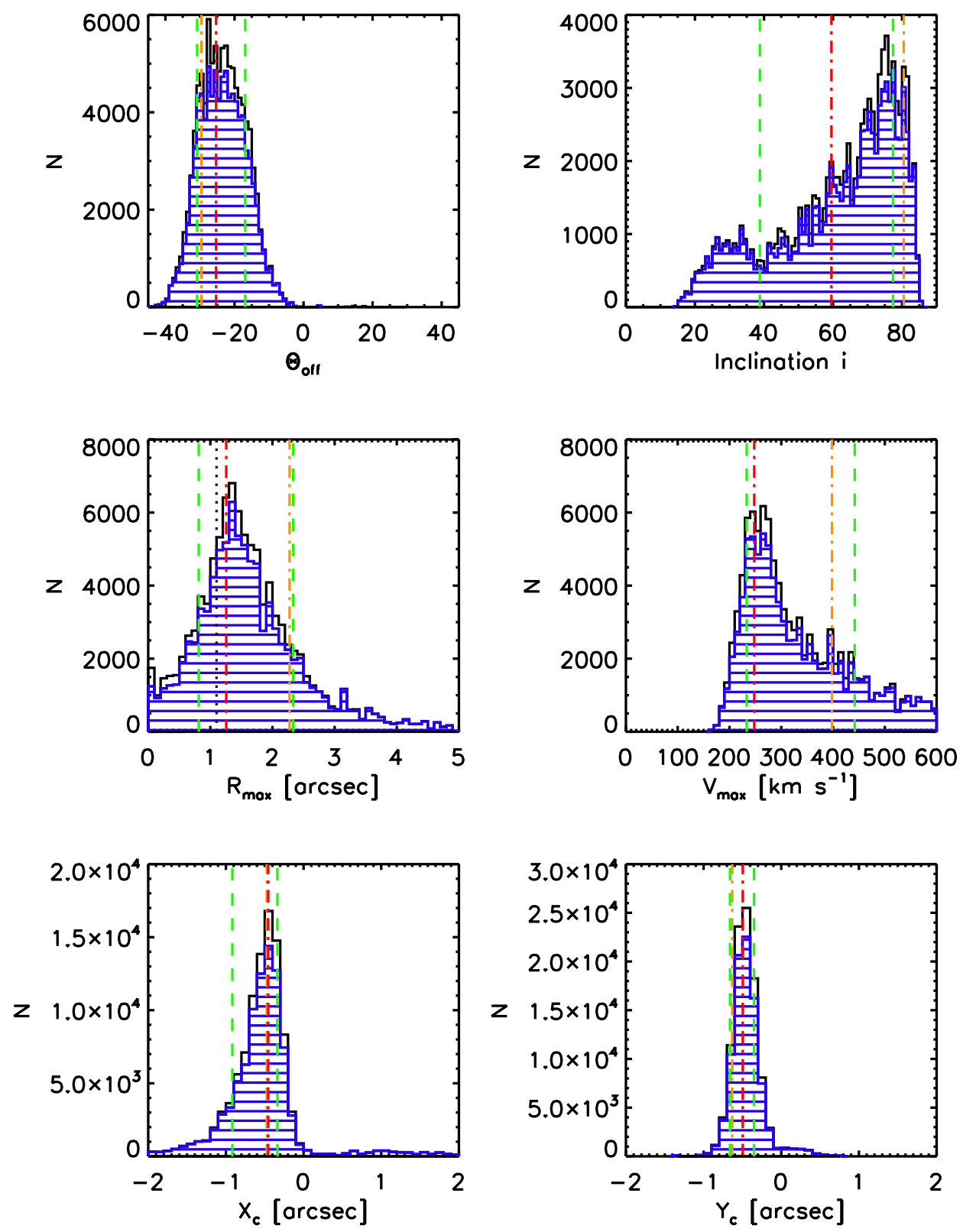

FIG. 13.- Posterior distributions derived using a Markov Chain Monte Carlo fitting approach and a simple thin disk model with six parameters: $\Theta_{o f f}$, the angle between the $\mathrm{PA}=52.44^{\circ}$ slit and the major axis of the disk (top left), $i$, the inclination of the disk relative to face-on (top right), $R_{\max }$, the radius at which the disk flattens to the maximum velocity (middle left), $V_{\max }$, the maximum velocity of the disk (middle right), and $X_{c}$ and $Y c$, the offsets of the slit crossover point relative to the disk center. The green dashed lines indicate the $67 \%$ confidence intervals quoted in Table 4 and the orange and red dot-dashed lines show the two random draws from the posterior distributions that are overplotted on the data in Figure 14 The black dotted line in the $R_{\max }$ panel corresponds to the maximum seeing during our spectroscopic observations. 

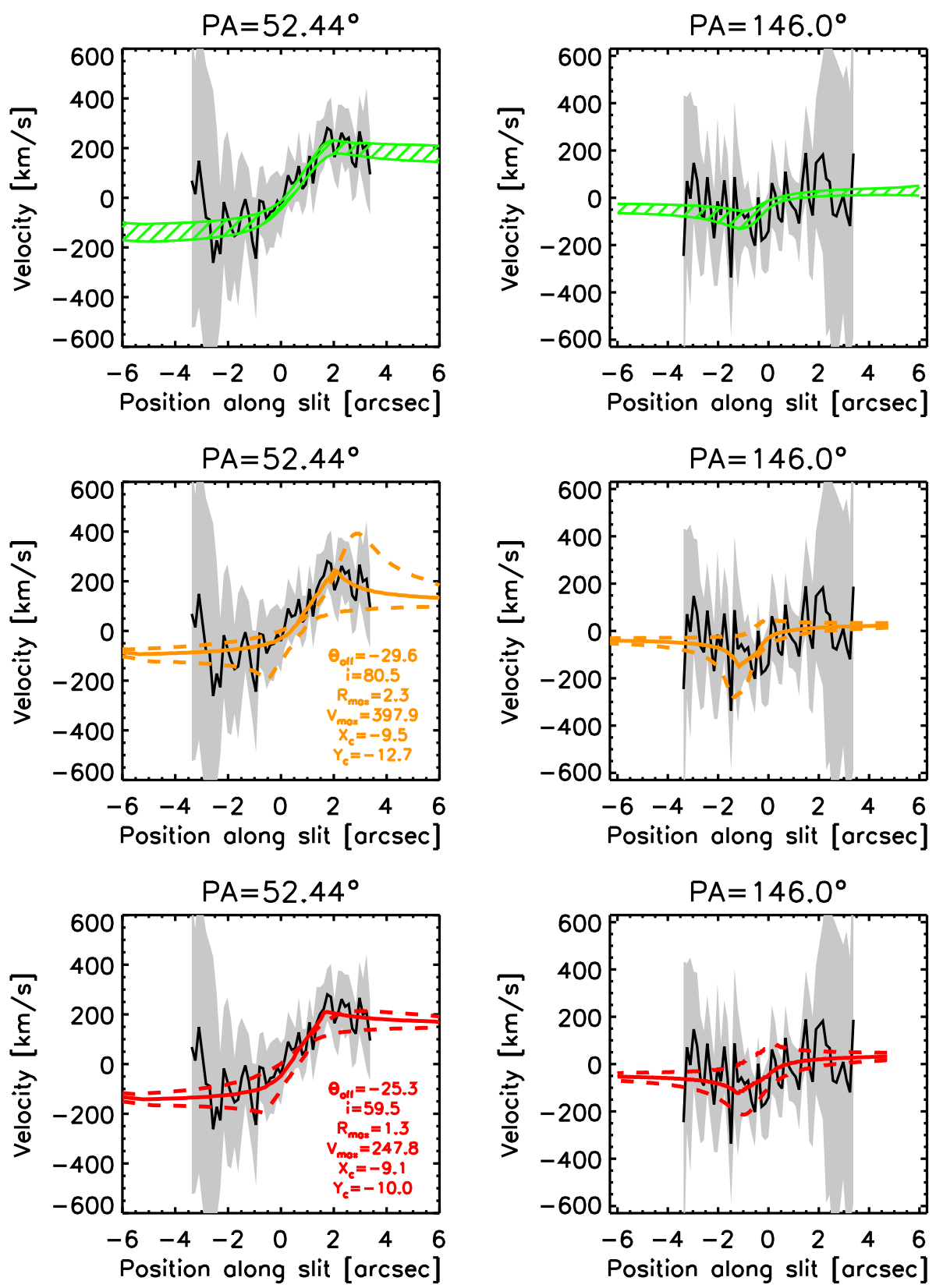

FIG. 14. - The observed HeII velocity profiles in the $\mathrm{PA}=52^{\circ} 44$ (left) and $\mathrm{PA}=146^{\circ} 0$ (right) slits are shown with a solid black line and grey shade regions indicating the error bars. Overplotted in color are velocity profile predictions from the thin disk toy model. The green hashed bands in the top row represent the range of models spanned by the $67 \%$ confidence intervals quoted in Table 4 The lower two rows show two random draws from the posterior distributions (orange and red colored lines), as indicated using the same color coding in Figure 13 The solid colored lines represent the velocity profile predicted along the center of the slit, while the dashed colored lines track the two slit edges. The corresponding model parameter values for each random draw are given in the left panel legend. 


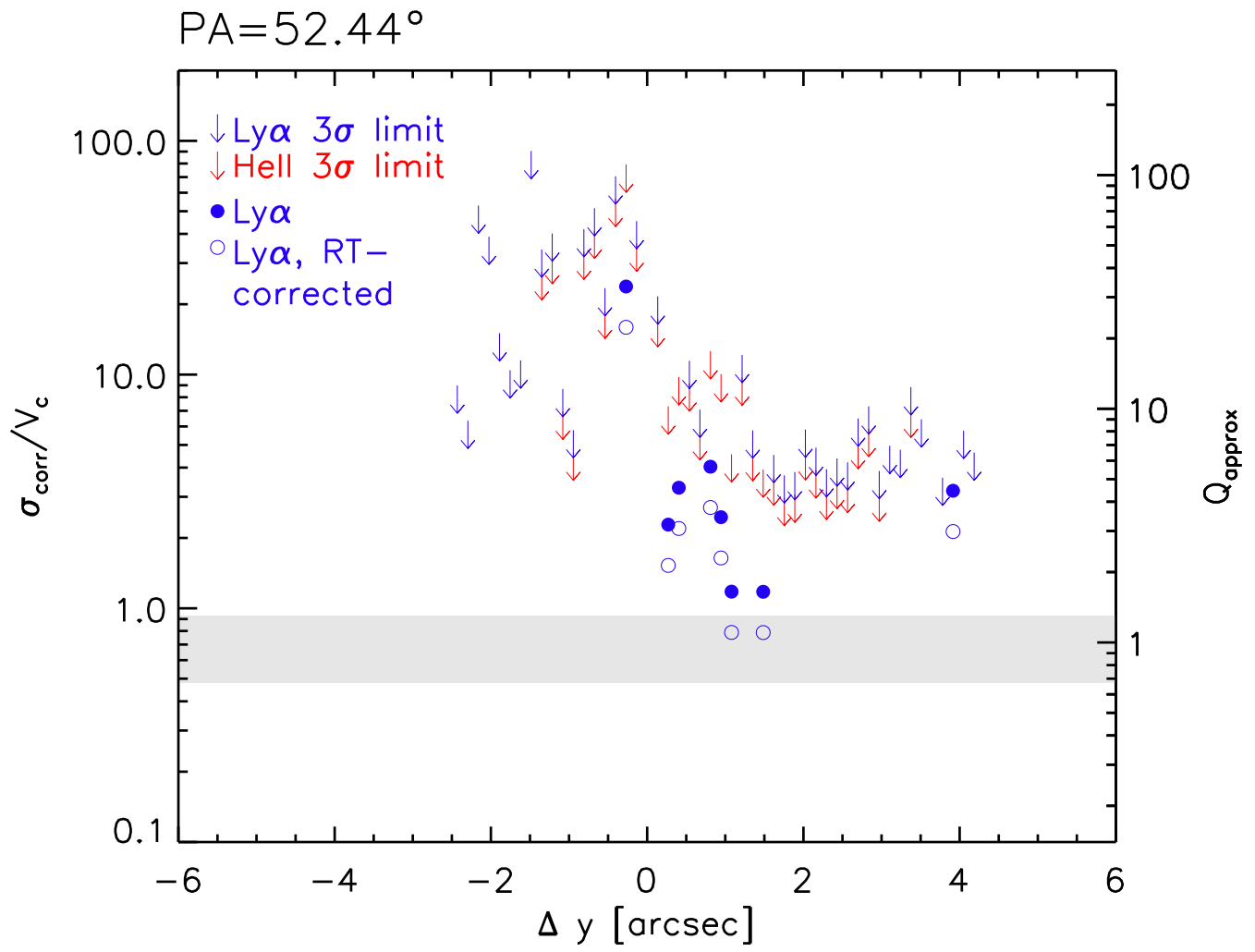

FIG. 15.- Measured $\sigma_{c o r r} / V_{c}$ as a function of position along the PA=52.44 slit, which coincides roughly the major axis of the proposed disk. Upper limits $(3 \sigma)$ are plotted for apertures where the measured linewidth is consistent with the instrumental resolution; otherwise the $\sigma_{\text {corr }}$ values are used, i.e., corrected for the instrumental resolution. $V_{c}$ is the measured velocity from the HeII line. The Ly $\alpha$ measurements are shown as solid circles, while the open blue circles represent the result of applying an approximate "radiative transfer correction," i.e., scaling the $\operatorname{Ly} \alpha \sigma_{\text {corr }}$ measurements such that the Ly $\alpha$ and HeII values agree in the one aperture along the PA=146. 0 slit where both lines are clearly resolved. The right axis gives the corresponding approximate Toomre $\mathrm{Q}$ values, under the assumption of a marginally stable disk. In this case, $Q_{\text {approx }} \approx(a / 1.4) \times\left(1.0 / f_{\text {gas }}\right) \times \sigma_{\text {corr }} / V_{c}$, following Genzel et al. $(2014)$, with $a$ being a geometric factor that can take values of $[1,1.4,2]$ for a Keplerian rotation curve, a flat rotation curve, and a solid-body rotation curve, respectively, and $f_{g a s}$ being the gas mass fraction. The grey-shading corresponds to the approximate values of $Q$ below which the gas is expected to be unstable to collapse. 